ARTICLE

\title{
Leptin receptor-expressing neuron Sh2b1 supports sympathetic nervous system and protects against obesity and metabolic disease
}

\author{
Lin Jiang ${ }^{1}$, Haoran Su${ }^{1}$, Xiaoyin $\mathrm{Wu}^{2}$, Hong Shen (10 ${ }^{1}$, Min-Hyun Kim¹, Yuan Li ${ }^{1}$, Martin G. Myers Jr (iD ${ }^{1,3}$, \\ Chung Owyang ${ }^{1,2} \&$ Liangyou Rui (i) ${ }^{1,2} \otimes$
}

Leptin stimulates the sympathetic nervous system (SNS), energy expenditure, and weight loss; however, the underlying molecular mechanism remains elusive. Here, we uncover Sh2b1 in leptin receptor (LepR) neurons as a critical component of a SNS/brown adipose tissue (BAT)/thermogenesis axis. LepR neuron-specific deletion of Sh2b1 abrogates leptinstimulated sympathetic nerve activation and impairs BAT thermogenic programs, leading to reduced core body temperature and cold intolerance. The adipose SNS degenerates progressively in mutant mice after 8 weeks of age. Adult-onset ablation of Sh2b1 in the mediobasal hypothalamus also impairs the SNS/BAT/thermogenesis axis; conversely, hypothalamic overexpression of human SH2B1 has the opposite effects. Mice with either LepR neuron-specific or adult-onset, hypothalamus-specific ablation of Sh2b1 develop obesity, insulin resistance, and liver steatosis. In contrast, hypothalamic overexpression of SH2B1 protects against high fat diet-induced obesity and metabolic syndromes. Our results unravel an unrecognized LepR neuron Sh2b1/SNS/BAT/thermogenesis axis that combats obesity and metabolic disease.

\footnotetext{
${ }^{1}$ Department of Molecular \& Integrative Physiology, University of Michigan Medical School, Ann Arbor, MI 48109, USA. ${ }^{2}$ Division of Gastroenterology and Hepatology, Department of Internal Medicine, University of Michigan Medical School, Ann Arbor, MI 48109, USA. ${ }^{3}$ Division of Metabolism and

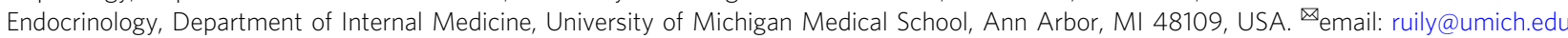


A dipose hormone leptin critically regulates body weight and metabolism, and disruption of leptin/leptin receptor (LepR) signaling results in morbid obesity and severe metabolic disease ${ }^{1}$. LepR is widely expressed in the hypothalamus, including the preoptic area (POA), lateral hypothalamus, dorsomedial hypothalamus (DMH), ventromedial hypothalamus, and arcuate nucleus (ARC) ${ }^{2,3}$. Leptin exerts its anti-obesity action by activating LepR signaling in hypothalamic energy balance circuits $^{4}$, but it remains elusive whether leptin regulates energy expenditure vs energy intake by similar or discrete pathways. Leptin signaling is mediated by tyrosine kinase JAK2 that interacts with long-form LepRb ${ }^{1}$. Of note, a number of negative regulators of JAK2, including SOCS3, PTP1B, RPTPe, and TCPTP, have been reported to promote obesity ${ }^{5-12}$, supporting the notion that JAK2 inhibitory molecules increase risk for leptin resistance, obesity, and metabolic disease. Interestingly, we identified a JAK2-binding protein Sh2b1 as a potent positive regulator of JAK2 (refs. 13,14); however, JAK2/Sh2b1 pathways in LepRexpressing neurons has not been explored in vivo.

$\mathrm{Sh} 2 \mathrm{~b} 1$ is an $\mathrm{SH} 2$ and $\mathrm{PH}$ domain-containing adaptor protein. It binds to JAK2 via its $\mathrm{SH} 2$ domain and robustly enhances JAK2 kinase activity ${ }^{14,15}$. Sh2b1 also binds to IRS1 and IRS2 and links JAK2 to IRS1/2-mediated activation of the PI 3-kinase pathway in cell cultures ${ }^{13}$. Aside from JAK2, Sh2b1 also binds to receptor tyrosine kinases, including insulin receptors, platelet-derived growth factor receptors, nerve growth factor receptor TrkA, and brain-derived neurotrophic factor (BDNF) receptor $\operatorname{TrkB}^{16-22}$. To examine Sh2b1 function in vivo, we generate and characterize global Sh2b1 knockout mice. Sh2b1-null mice develop severe leptin resistance, obesity, and type 2 diabetes $^{23,24}$. We and other groups further demonstrate that the metabolic function of Sh2b1 has conserved from flies to humans. Deletion of $S h 2 b$ results in fat accumulation in Drosophila ${ }^{25}$. In human genome-wide association studies, numerous $S H 2 B 1$ single-nucleotide polymorphisms (SNPs) have been identified to link to obesity, type 2 diabetes, and cardiovascular diseases ${ }^{26-28}$. Deletion of chromosomal 16p11.2, which encompasses the $S H 2 B 1$ gene, is associated with severe obesity in humans ${ }^{29-32}$. Human $S H 2 B 1$ missense mutations are cosegregated with the obesity and metabolic disease traits ${ }^{33-38}$. Thus, SH2B1 is emerging as a critical regulator of body weight and metabolism in both animals and humans; however, SH2B1 target cell types remain poorly understood.

We report that neuron-specific restoration of Sh2b1 expression reverses the obesity phenotypes of Sh $2 b 1$-null mice ${ }^{39}$, indicating that neurons mediate Sh2b1 actions on body weight and metabolism. Given that Sh2b1 augments LepRb/JAK2 signaling in cell cultures, we postulate that Sh2b1 might cell-autonomously increase the ability of LepR neurons to control energy balance and body weight, perhaps by directly enhancing leptin signaling. In this study, we generate and characterize LepR cell-specific $\operatorname{Sh} 2 b 1$ knockout $\left(\operatorname{Sh} 2 b 1^{\Delta L e p R}\right)$ mice. Sh $2 b 1^{\Delta L e p R}$ mice, like global $S h 2 b 1$ knockout mice, develop obesity, insulin resistance, and liver steatosis. Remarkably, Sh2b1 deficiency in LepR neurons abrogates the ability of leptin to stimulate sympathetic nerves innervating brown adipose tissue (BAT), leading to BAT dysfunction and reduced core body temperature in $\operatorname{Sh} 2 b 1^{\Delta L e p R}$ mice. Collectively, our results unveil an unrecognized leptin/Sh2b1/ sympathetic nerve/adipose thermogenesis axis that combats obesity, type 2 diabetes, and liver steatosis.

\section{Results}

Sh2b1 ${ }^{\Delta \text { LepR }}$ mice spontaneously develop obesity. To determine the role of Sh2b1 in LepR neurons, we generated Sh $2 b 1^{\Delta L e p R}$ mice (Sh2b1f/f;LepR-Cre ${ }^{+/+}$) by crossing Sh2b1/ff mice with LepR-Cre drivers. LepR-Cre mice were characterized previously ${ }^{40,41}$. Mice were in a C57BL/6J background and fed a standard chow diet. $\operatorname{Sh} 2 b 1^{\triangle L e p R}$ male and female mice progressively became heavier than sex/age-matched Sh2b1f/f and LepR-Cre mice (Fig. 1a). Fat content was dramatically higher in $\operatorname{Sh} 2 b 1^{\Delta L e p R}$ males and females relative to sex/age-matched $S h 2 b 1^{f / f}$ and LepR-Cre mice (Fig. 1b). Both gonadal and inguinal white adipose tissue (WAT) depots were significantly larger in $S h 2 b 1^{\Delta L e p R}$ relative to $S h 2 b 1^{f / f}$ and LepR-Cre mice (Supplementary Fig. 1a). Individual white adipocyte size was substantially larger in $S h 2 b 1^{\Delta L e p R}$ than in LepR-Cre mice (Fig. 1c). Lean mass was not significantly different between $S h 2 b 1^{\Delta L e p R}$ and LepR-Cre mice (Supplementary Fig. 1b). To gain insight into the underlying mechanism, we analyzed energy balance. Food intake was relatively normal (Fig. 1d). $\mathrm{O}_{2}$ consumption and $\mathrm{CO}_{2}$ production (per mouse) were also not significantly different between $S h 2 b 1^{\Delta L e p R}$ and $S h 2 b 1^{f / f}$ mice (Fig. 1e, Supplementary Fig. 1c). Of note, $\mathrm{O}_{2}$ consumption and $\mathrm{CO}_{2}$ production, after normalization to body weight, were significantly lower in $S h 2 b 1^{\Delta L e p R}$ males and females relative to sex/age-matched Sh2b1f/f mice (Supplementary Fig. 1d, e). Core body temperature was significantly lower in $S h 2 b 1^{\Delta L e p R}$ males and females relative to sex/age-matched LepR-Cre or Sh $2 b 1^{f / f}$ mice (Fig. 1f, g). We further confirmed that $S h 2 b 1^{\Delta L e p R}$ mice had lower body temperature using E-Mitters, and $S h 2 b 1^{\Delta L e p R}$ locomotor activity was relatively normal (Fig. $1 \mathrm{~h}$ ). These data indicate that Sh2b1 in LepR neurons is indispensable for the maintenance of both body weight and core body temperature.

Sh2b1 ${ }^{\Delta L e p R}$ mice develop insulin resistance and liver steatosis. Obesity promotes type 2 diabetes and nonalcoholic fatty liver disease (NAFLD), prompting us to assess insulin sensitivity and hepatic lipid content. Sh $2 b 1^{\triangle L e p R}$ males and females developed hyperglycemia and hyperinsulinemia compared to sex/age-matched LepR-Cre mice at 19-20 weeks of age (Fig. 2a). In glucose (GTT) or insulin (ITT) tolerance tests, blood glucose levels were markedly higher in $S h 2 b 1^{\Delta L e p R}$ males and females relative to sex/ age-matched LepR-Cre or Sh2b1 f/f mice (Fig. 2b). Consistently, insulin-stimulated phosphorylation of Akt (pThr308 and pSer473) in liver and skeletal muscle was substantially lower in $S h 2 b 1^{\Delta L e p R}$ than in LepR-Cre mice (Fig. 2c). Sh $2 b 1^{\Delta L e p R}$ mice also developed severe liver steatosis, as demonstrated by markedly increased lipid droplet number and size and triacylglycerol (TAG) levels in the liver (Fig. 2d, e). These results suggest that Sh2b1 in LepR neurons combats against insulin resistance, type 2 diabetes, and NAFLD.

Adult-onset ablation of hypothalamic $S h 2 b 1$ results in obesity. We recently reported that neuronal Sh2b1 promotes brain development ${ }^{16}$. To distinguish between brain development-dependent and -independent actions of Sh2b1 on body weight and metabolism, we generated adult-onset, hypothalamus-specific Sh2b1 knockout mice by bilaterally microinjecting AAV1-hSyn-Cre vectors into the mediobasal hypothalami $(\mathrm{MBH})$ of $S h 2 b 1^{f / f}$ males at 10 weeks of age. AAV1-hSyn-green fluorescent protein (GFP) vectors were used as control. Bilateral $\mathrm{MBH}$ injections were histologically verified (Supplementary Fig. 2a). MBH-specific ablation of Sh2b1 substantially increased body weight and fat content (Fig. 3a, b). As an additional control, AAV1-hSyn-Cre vectors were bilaterally injected into the MBH of wild-type C57BL/6 males. There was no difference in body weight and fat content between the AAV1-hSyn-Cre and AAV1-hSyn-GFP groups (Supplementary Fig. 2b-c). $\mathrm{O}_{2}$ consumption and $\mathrm{CO}_{2}$ production (per mouse) were not significantly different between the AAV1-hSyn-Cre and AAV1-hSyn-GFP groups (Fig. 3c). Nonetheless, $\mathrm{O}_{2}$ consumption and $\mathrm{CO}_{2}$ production, after normalization to body weight, were significantly lower in AAV1-hSyn-Cre relative to AAV1-hSyn-GFP groups in the dark 


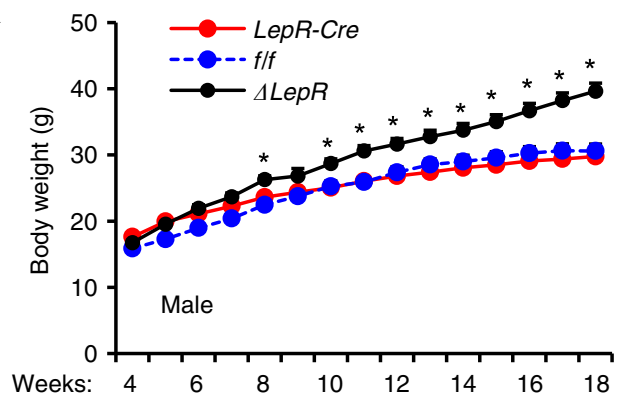

b
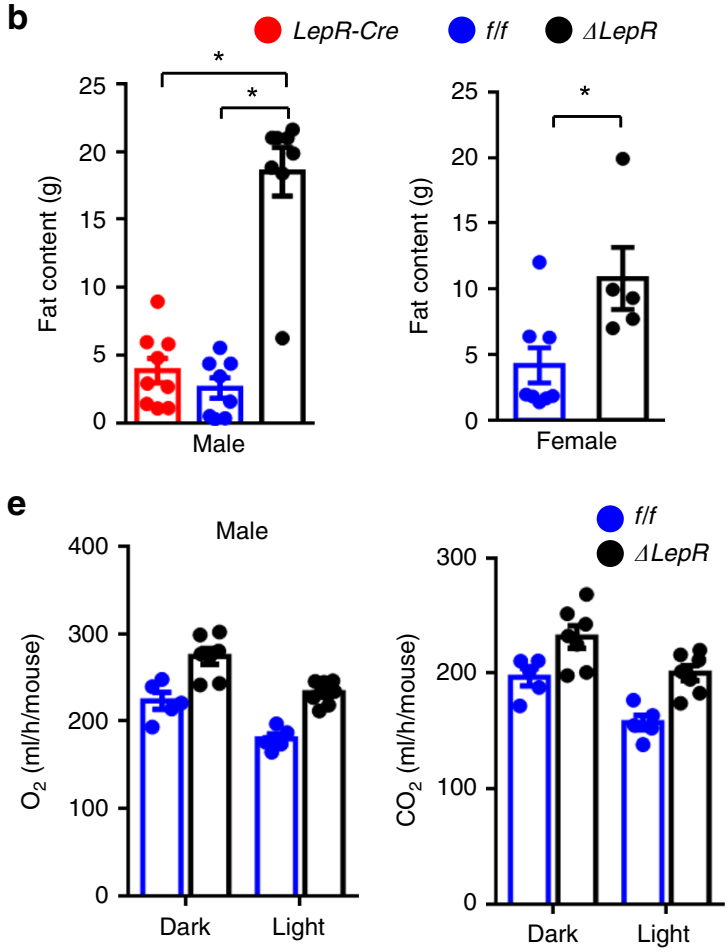
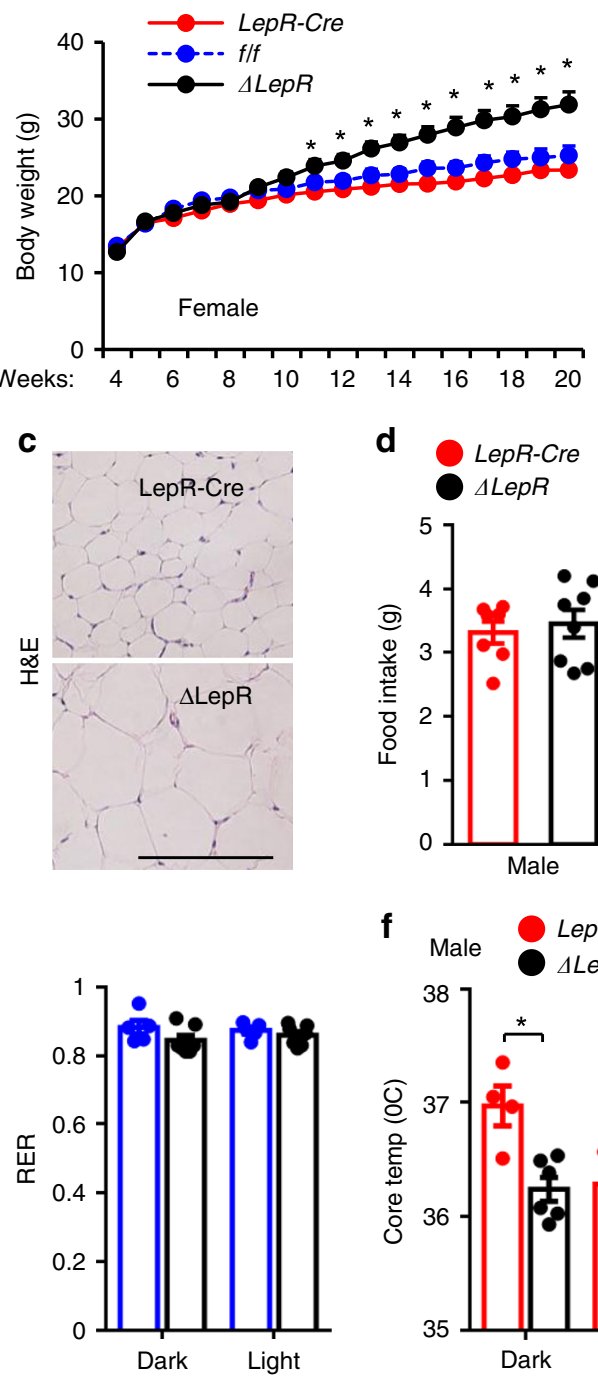

C

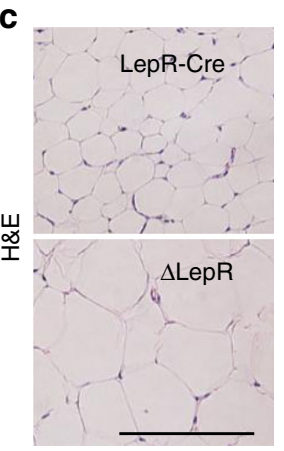

d LepR-Cre

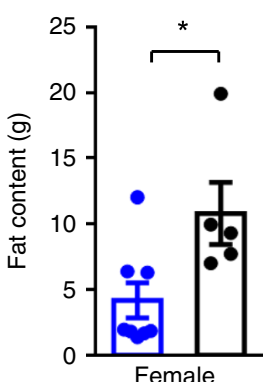

g

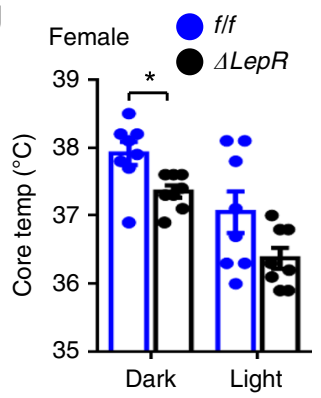

h

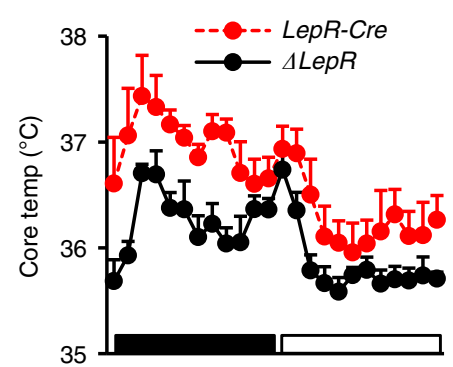

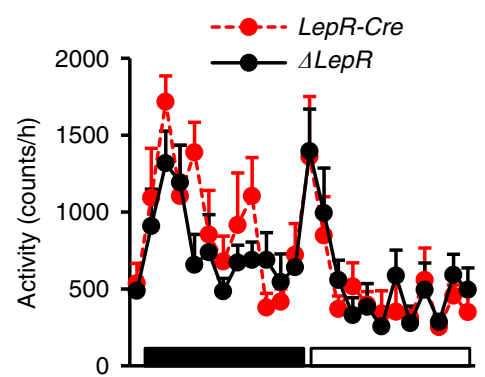

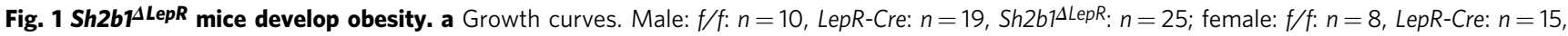

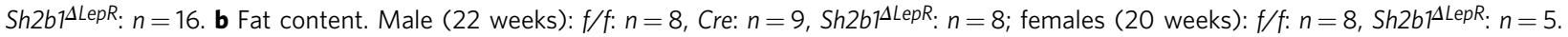

c Representative H\&E staining of epididymal WAT sections at 22 weeks of age ( 3 pairs). Scale bar: $200 \mu$ m. $\mathbf{d}$ Food intake of males at 10 weeks of age.

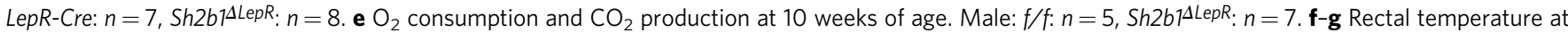

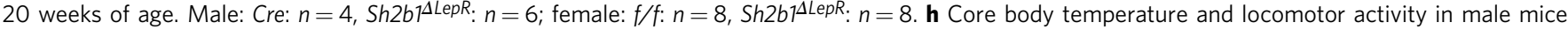
(10 weeks) recorded using pre-implanted E-Mitters. LepR-Cre: $n=4$, Sh2b74LepR: $n=6$. Data are presented as mean \pm SEM. ${ }^{*} p<0.05$, two-tailed unpaired Student's t-test (b female, $\mathbf{d}-\mathbf{g}$ ), two-way ANOVA (a), or one-way ANOVA (b male). Source data are provided as a Source Data file.

phase (Supplementary Fig. 2d). Core body temperature was significantly lower in the AAV1-hSyn-Cre mice in the dark cycle (Fig. 3d). Notably, MBH-specific ablation of Sh2b1 significantly increased food intake (Fig. 3e). Like Sh $2 b 1^{\Delta L e p R}$ mice, adult-onset and MBH-specific Sh2b1 knockout mice developed hyperinsulinemia, glucose intolerance, and insulin resistance (Fig. $3 \mathrm{f}, \mathrm{g}$ ).
Insulin-stimulated phosphorylation of hepatic Akt was lower in AAV1-hSyn-Cre-transduced than in AAV1-hSyn-GFP-transduced Sh2b1f/f mice (Fig. 3h). MBH-specific Sh2b1 knockout mice also developed severe liver steatosis, as demonstrated by elevated levels of hepatocyte lipid droplets (Oil Red O staining of liver sections) and high levels of liver TAG (Fig. 3i). These data indicate that Sh2b1 in 
a
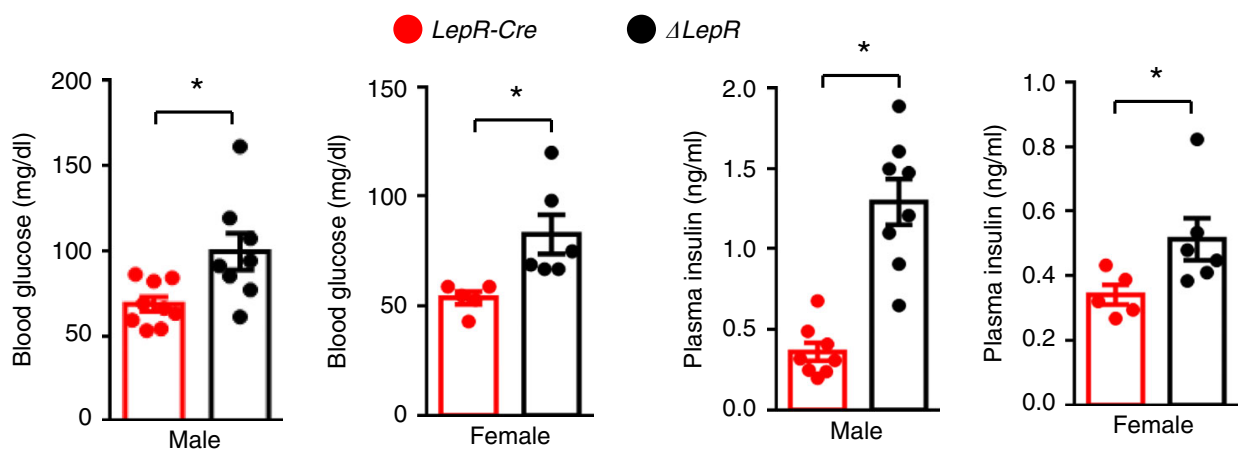

b
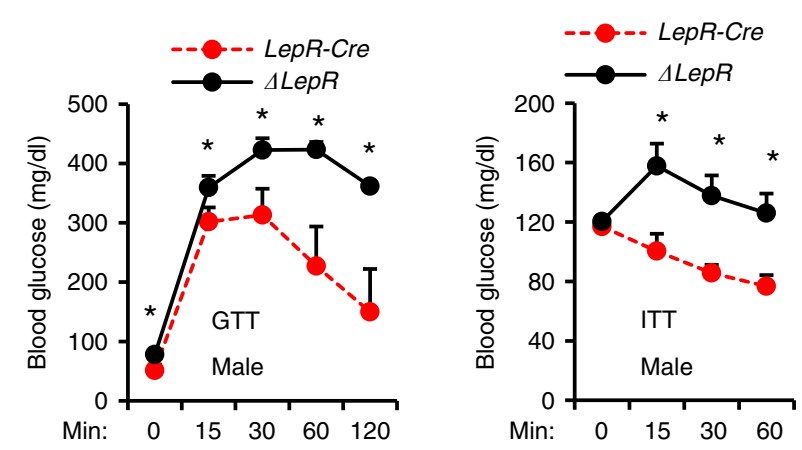

d Male
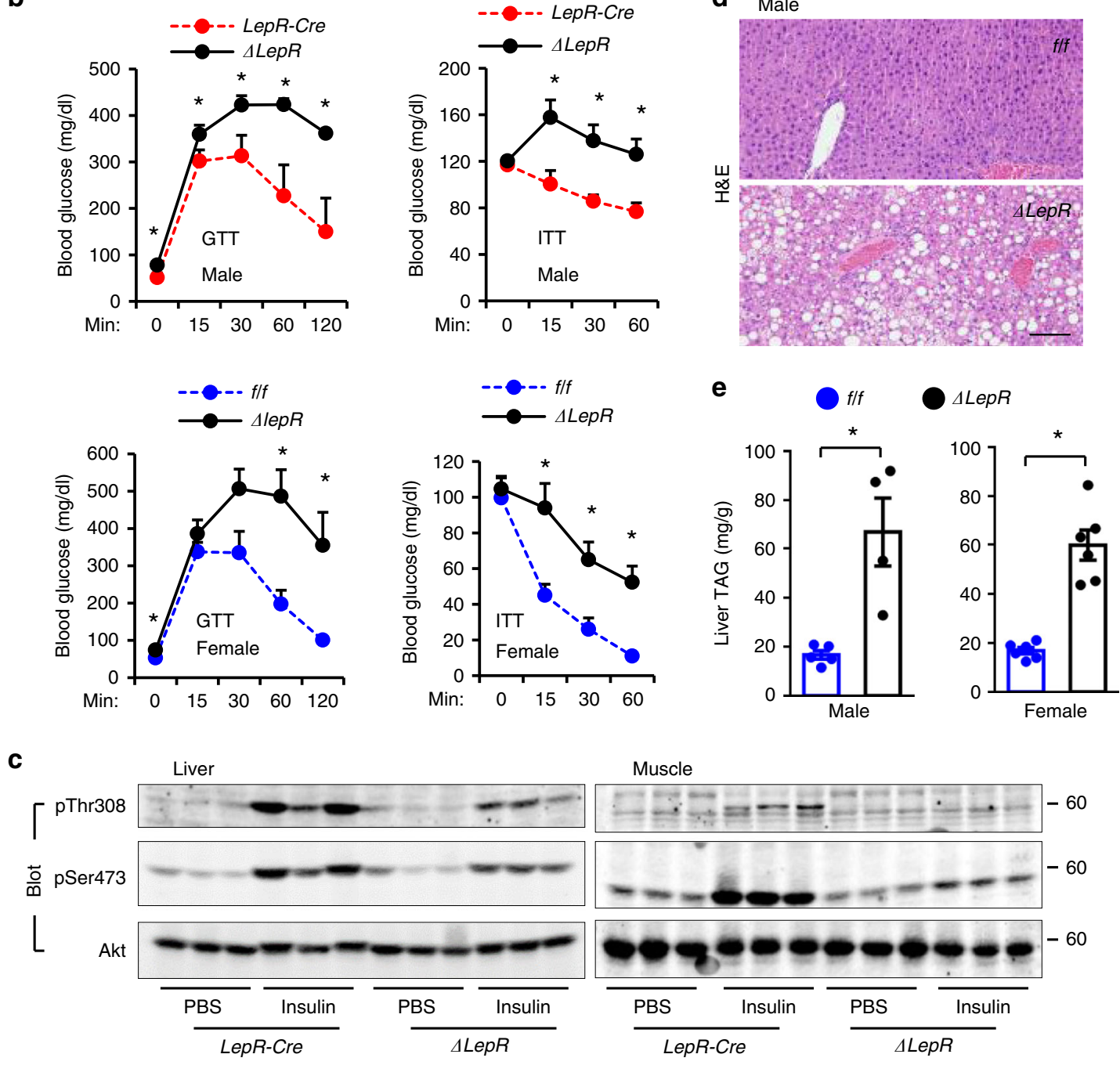

Fig. 2 Sh2b7 ${ }^{\Delta L e p R}$ mice develop insulin resistance, glucose intolerance, and liver steatosis. a Overnight fasting blood glucose and insulin levels at

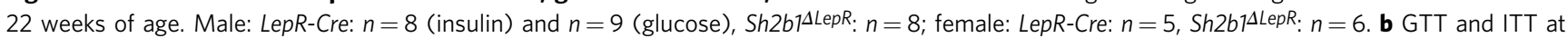
20 weeks of age. Male: LepR-Cre: $n=9$, Sh2b1 $1^{\Delta L} R: n=8$; female: $f / f: n=5$, Sh2b14LepR: $n=5$. c Male mice (23 weeks) were stimulated with insulin. Liver and skeletal muscle extracts were immunoblotted with the indicated antibodies. Male: $f / f: n=6$, Sh2b1LLepR: $n=6$; female: $f / f: n=6$, Sh2b1 $\operatorname{LLepR}: n=6$. d Representative H\&E staining of liver sections at 23 weeks of age (3 pairs). Scale bar: $200 \mu \mathrm{m}$. e Liver TAG levels (normalized to liver weight) at 23 weeks of age. Male: $f / f: n=5, \operatorname{Sh} 2 b 7^{\Delta L e p R}: n=4$; female: $f / f: n=6$, Sh $2 b 7^{\Delta L e p R}: n=6$. Data are presented as mean \pm SEM. ${ }^{*} p<0.05$, two-tailed unpaired Student's $t$-test. Source data are provided as a Source Data file.

the $\mathrm{MBH}$ regulates body weight, metabolism, food intake, and/or body temperature independently of its action on brain development.

Hypothalamic overexpression of SH2B1 ameliorates obesity. To determine whether MBH-specific overexpression of human SH2B1 protects against obesity, AAV9-CAG-SH2B1 or AAV9-
CAG-GFP (control) vectors were bilaterally injected into the $\mathrm{MBH}$ of $\mathrm{C} 57 \mathrm{BL} / 6 \mathrm{~J}$ males. Mice were fed an HFD to induce obesity. Recombinant SH2B1 was detected in AAV9-CAGSH2B1-transduced but not AAV9-CAG-GFP-transduced mice (Supplementary Fig. 3a, b). Body weight and fat content were significantly lower in the AAV9-CAG-SH2B1 group relative to 
a

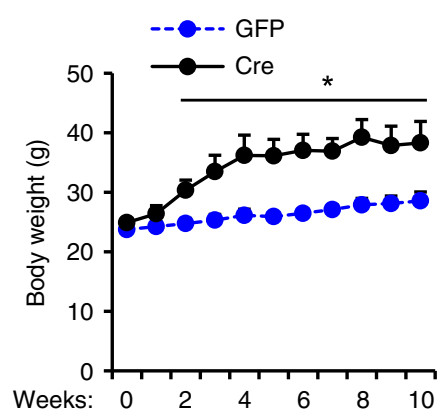

b

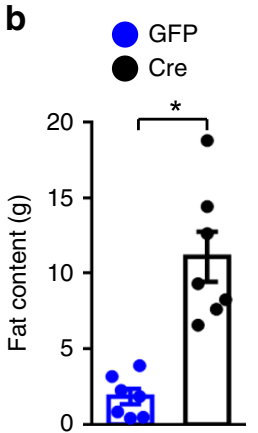

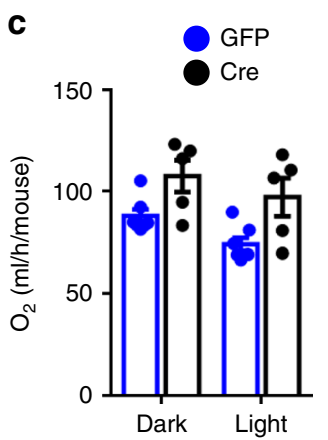

d

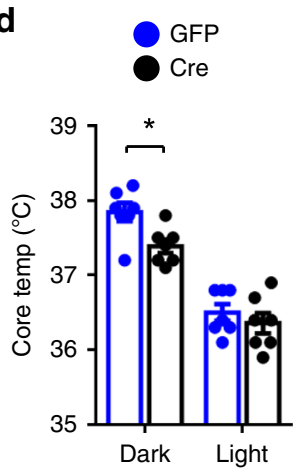

$\mathbf{h}$

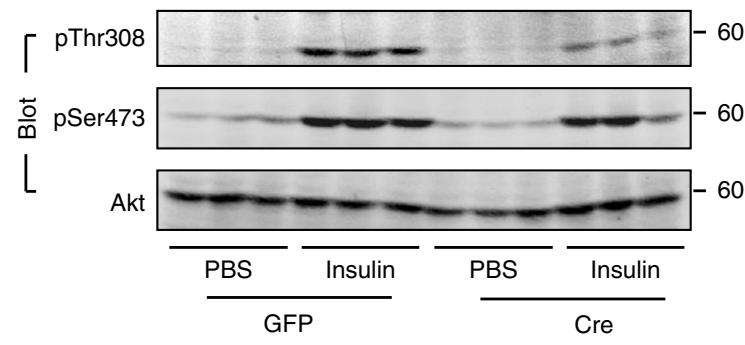

e

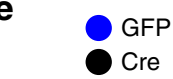

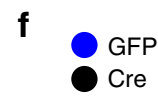

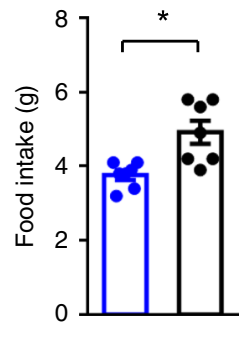

g
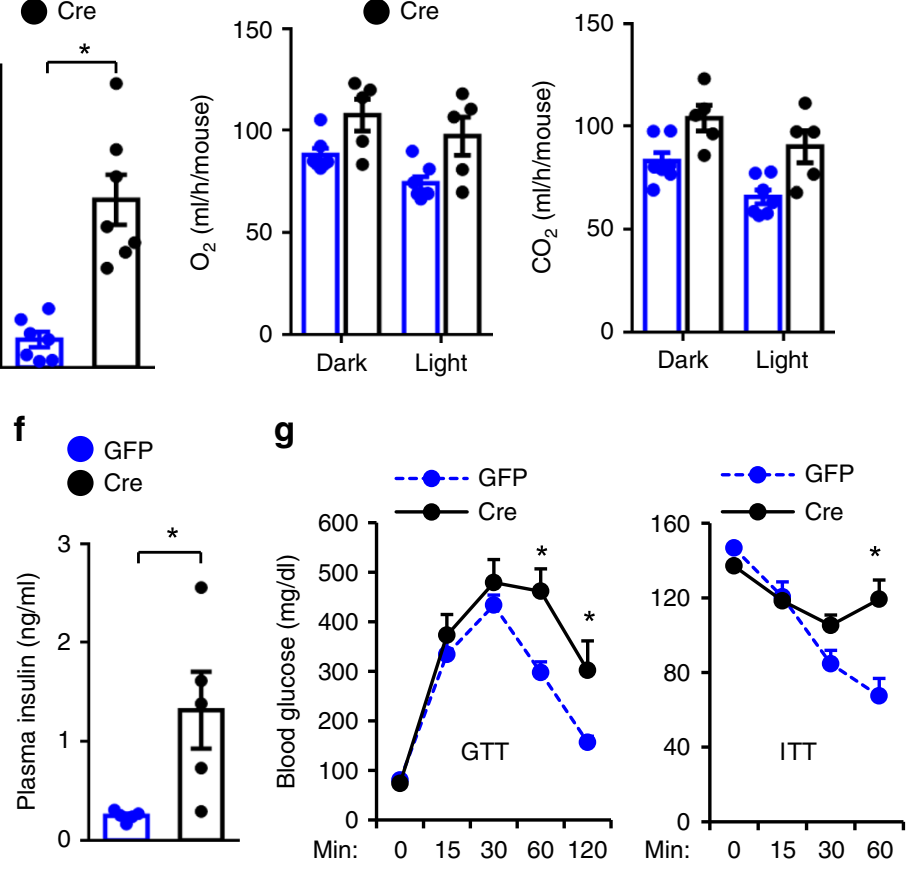

i
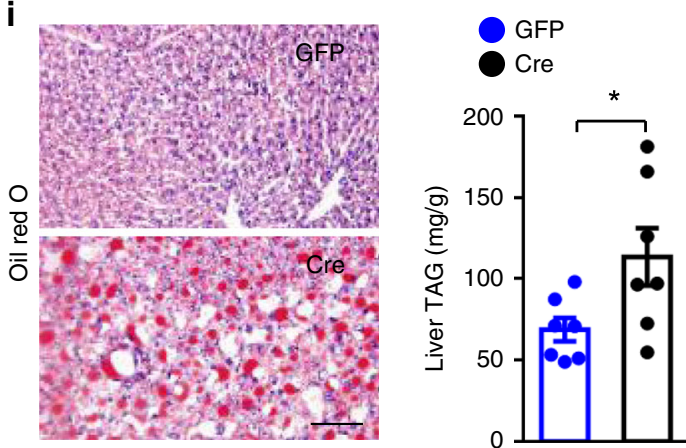

Fig. 3 Adult-onset, MBH-specific ablation of Sh2b1 results in obesity, insulin resistance, and liver steatosis. AAV1-hSyn-Cre or AAV1-hSyn-GFP vectors were bilaterally injected into the $\mathrm{MBH}$ of $\mathrm{Sh} 2 \mathrm{~b} \mathrm{f}^{\mathrm{f} / \mathrm{f}}$ males at 10 weeks of age. a Growth curves. b Fat content in 10 weeks post transduction. c Energy expenditure in 10 weeks after transduction. $\mathbf{d}$ Rectal temperature in 10 weeks following transduction. e Food intake at 10 weeks post transduction. f Overnight fasting plasma insulin levels in 10 weeks after transduction. $\mathbf{g}$ GTT and ITT in 10-11 weeks post transduction. $\mathbf{h}$ Mice were stimulated with insulin in 11 weeks post transduction. Liver extracts were immunoblotted with the indicated antibodies $(n=3$ mice per group). i Representative Oil Red O staining of liver sections and liver TAG levels (normalized to liver weight) in 11 weeks post transduction. a, b, d, e, g, i: AAV1-hSyn-GFP: $n=7$, AAV1hSyn-Cre: $n=7$; c: AAV1-hSyn-GFP: $n=7$, AAV1-hSyn-Cre: $n=5$; f: AAV1-hSyn-GFP: $n=5$, AAV1-hSyn-Cre: $n=5$. Scale bar: $100 \mu$ m. Data are presented as mean \pm SEM. ${ }^{\star} p<0.05$, two-tailed unpaired Student's $t$-test. Source data are provided as a Source Data file.

the AAV9-CAG-GFP group (Fig. 4a, b). $\mathrm{O}_{2}$ consumption and $\mathrm{CO}_{2}$ production (per mouse) were not significantly different between the GFP and SH2B1 groups (Fig. 4c). Upon normalization to body weight, $\mathrm{O}_{2}$ consumption and $\mathrm{CO}_{2}$ production were significantly higher in the AAV9-CAG-SH2B1 group relative to the AAV9-CAG-GFP group (Supplementary Fig. 3c). Core body temperature was significantly higher in the AAV9CAG-SH2B1 group (Fig. 4d). MBH-specific overexpression of SH2B1 substantially ameliorated HFD-induced insulin resistance and glucose intolerance, as assessed by ITT, GTT, and insulin-stimulated phosphorylation of Akt (Fig. 4e, f). Overexpression of SH2B1 in the hypothalamus also blocked HFDinduced liver steatosis, as demonstrated by a marked reduction in lipid droplet number and size and liver TAG levels in the AAV9-CAG-SH2B1 relative to AAV9-CAG-GFP groups (Fig. 4g). These data further confirm that hypothalamic Sh2b1, perhaps in LepR neurons, protects against obesity, type 2 diabetes, and NAFLD in adult mice.

LepR neuron Sh2b1 is required for brown fat thermogenesis. Given that BAT and beige fat promote adaptive thermogenesis, energy expenditure, and weight loss, we examined the impact of hypothalamic Sh2b1 on BAT activity. Ablation of Sh2b1 in either LepR neurons (Sh2b1 $1^{\Delta L e p R}$ mice) or the MBH (AAV1-hSyn-Cretransduced $S h 2 b 1^{f / f}$ mice) caused whitening of BAT (e.g. enlarged lipid droplets) and dramatic downregulation of uncoupling protein 1 (Ucp1) (Fig. 5a). Ucp1 protein and mRNA were barely detectable in Sh2b1 $1^{\Delta L e p R}$ mice at 22 weeks of age (Fig. 5b, c). Ucp1 expression in inguinal WAT markedly decreased in $S h 2 b 1^{\Delta L e p R}$ mice (Supplementary Fig. 1g). Of note, absolute expression levels of Ucp1 was markedly higher in BAT than in WAT. Conversely, MBH-specific overexpression of SH2B1 reversed HFD-induced 
a

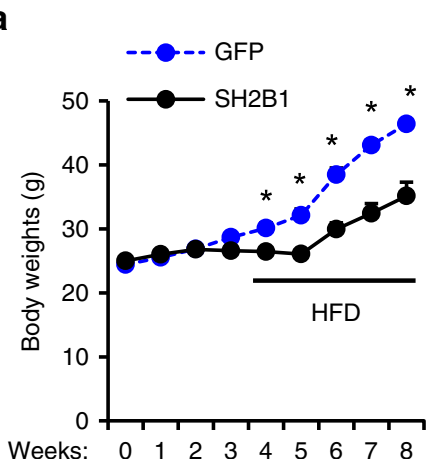

d

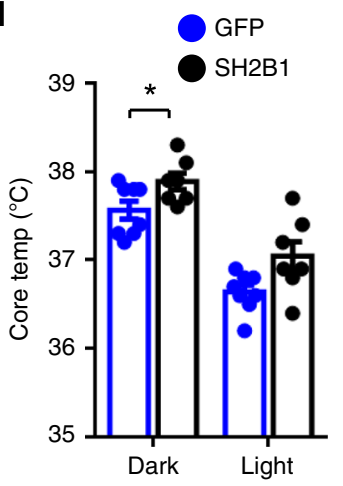

b

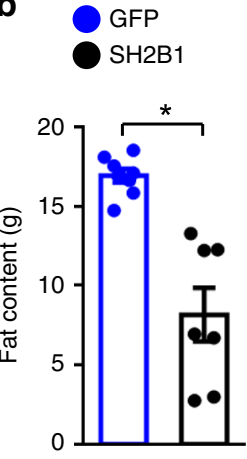

C

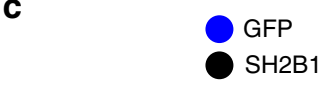

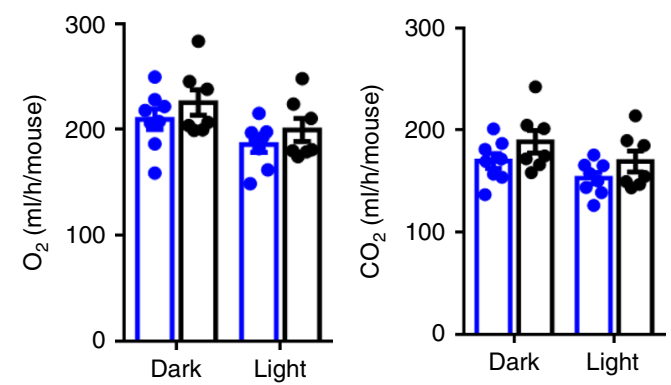

e
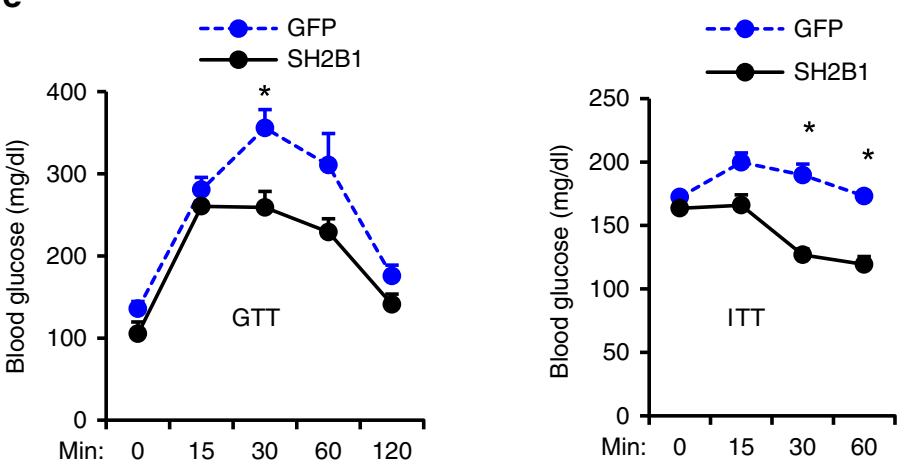

f
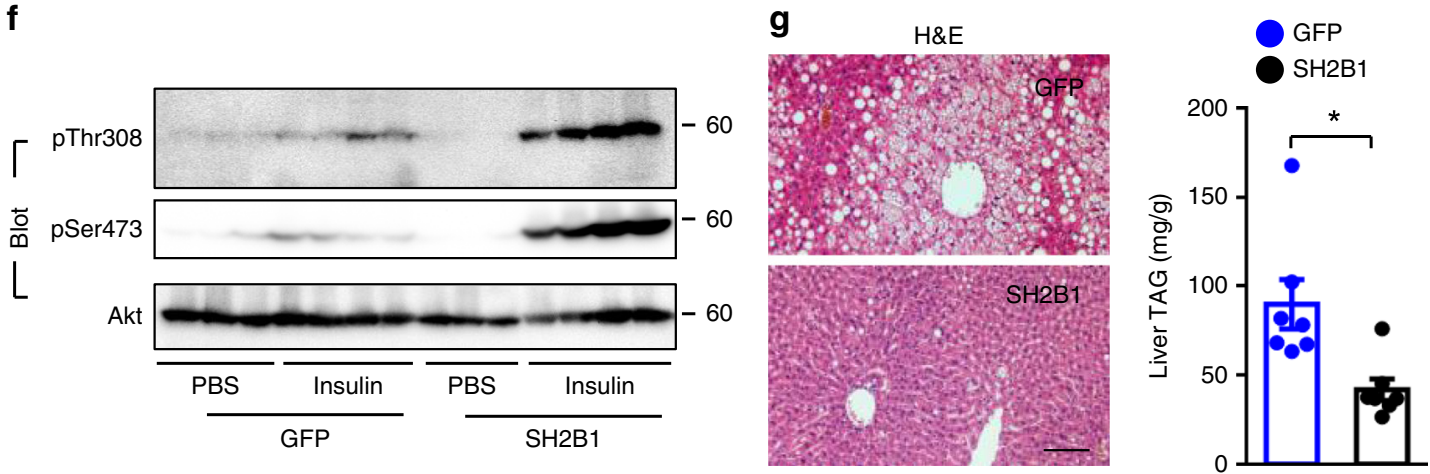

Fig. 4 MBH-specific overexpression of SH2B1 protects against HFD-induced obesity, insulin resistance, and liver steatosis. AAV9-CAG-SH2B1 $\beta(n=$ 7) or AAV9-CAG-GFP ( $n=7-8)$ vectors were bilaterally injected into the MBH of adult C57BL/6J males, followed by HFD feeding. a Growth curves. b Fat content in 10 weeks post HFD. $\mathbf{c}$ Energy expenditure in 10 weeks post HFD. $\mathbf{d}$ Rectal temperature in 10 weeks post HFD. e GTT and ITT in 10-11 weeks post HFD. $\mathbf{f}$ Mice (11 weeks post HFD) were stimulated with PBS ( $n=3$ mice per group) or insulin ( $n=4$ mice per group). Liver extracts were immunoblotted with the indicated antibodies. $\mathbf{g}$ Representative H\&E staining of liver sections and liver TAG levels (normalized to liver weight) in 12 weeks post HFD. Scale bar: $200 \mu \mathrm{m}$. Data are presented as mean \pm SEM. ${ }^{*} p<0.05$, two-tailed unpaired Student's $t$-test. Source data are provided as a Source Data file.

whitening of BAT and downregulation of Ucp1 (Fig. 5a). Ucp1 protein and mRNA levels were substantially higher in AAV9CAG-SH2B1-transduced relative to AAV9-CAG-GFP-transduced mice (Fig. 5b, c). Ucp1 mediates adaptive thermogenesis and energy expenditure ${ }^{42}$. Accordingly, Sh $2 b 1^{\Delta L e p R}$ mice displayed markedly lower core body temperature compared to LepR-Cre mice upon cold exposure (on a chow diet), and mice with $\mathrm{MBH}$ specific overexpression of SH2B1 (on a HFD) had the opposite effects (Fig. 5d). Thus, we uncovered an unrecognized hypothalamic Sh2b1/BAT axis that critically regulates adaptive thermogenesis and core body temperature.

LepR neuron Sh2b1 mediates leptin stimulation of the SNS. Considering the pivotal role of the sympathetic nervous system (SNS) in BAT activation, we assessed the impact of Sh2b1 deficiency on the ability of leptin to stimulate sympathetic nerve transmissions in BAT. Both male and female Sh $2 b 1^{\Delta L e p R}$ mice developed hyperleptinemia (Fig. 6a). Leptin stimulated phosphorylation of hypothalamic Stat 3 to a lower degree in $S h 2 b 1^{\Delta L e p R}$ relative to $S h 2 b 1^{f / f}$ mice (Fig. 6b, c). Conversely, MBH-specific overexpression of $\mathrm{SH} 2 \mathrm{~B} 1$ augmented leptin-stimulated phosphorylation of hypothalamic Stat3 (Fig. 6b). To directly assess sympathetic nerve activity (SNA), we electrophysiologically recorded BAT SNA in $S h 2 b 1^{\Delta L e p R}$ mice at both 6 weeks (prior to the onset of obesity) and 12 weeks of age. Baseline SNA was significantly lower in $S h 2 b 1^{\Delta L e p R}$ relative to $S h 2 b 1^{f / f}$ mice (Fig. 6d, e). In agreement with the previous reports ${ }^{43}$, central injection of leptin progressively and markedly increased SNA in wild type (i.e. $\operatorname{Sh} 2 b 1^{f / f}$ ) mice (Fig. 6d, f). It is likely that multiple synaptic modifications and/or polysynaptic transmissions contribute to a delayed onset of the leptin action on SNA. Strikingly, deletion of 
a

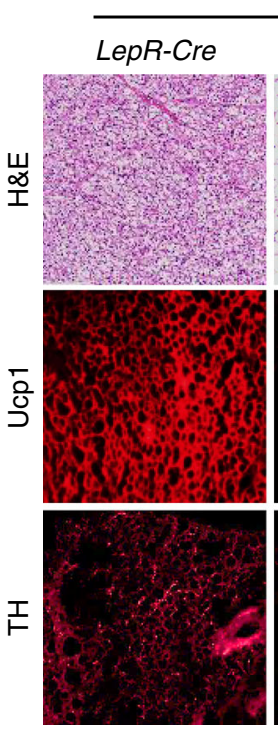

Chow

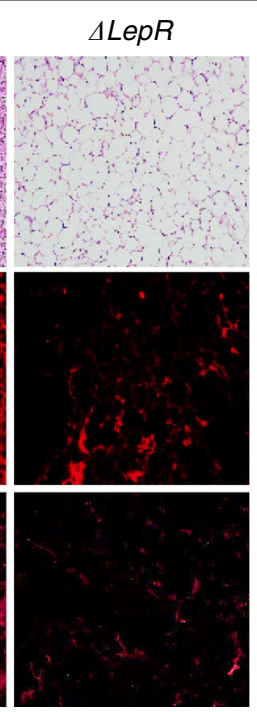

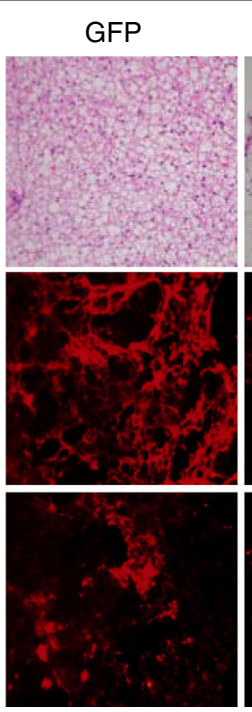

Cre
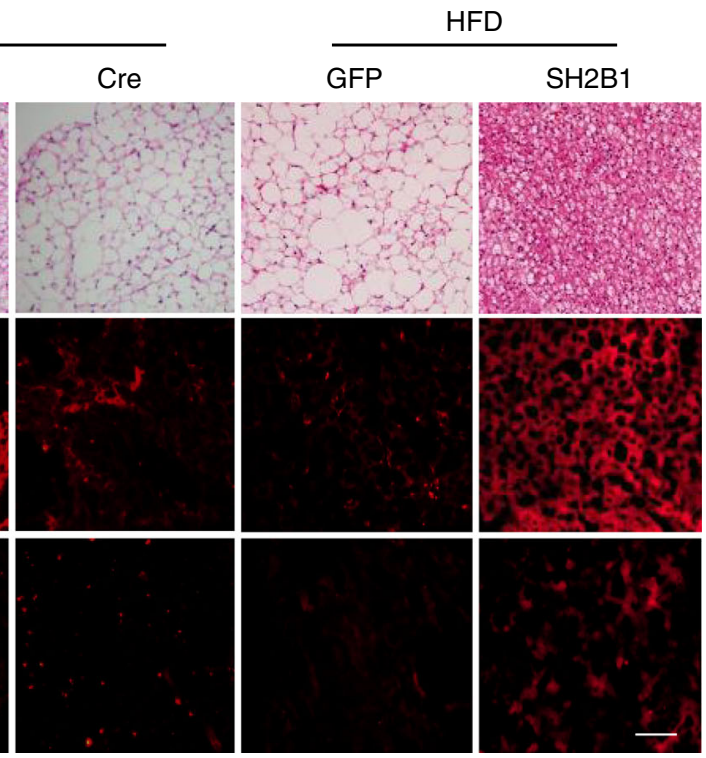

b
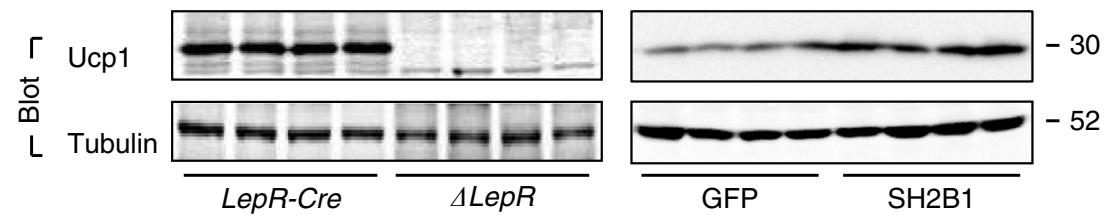

C

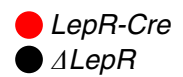

GFP
SH2B1
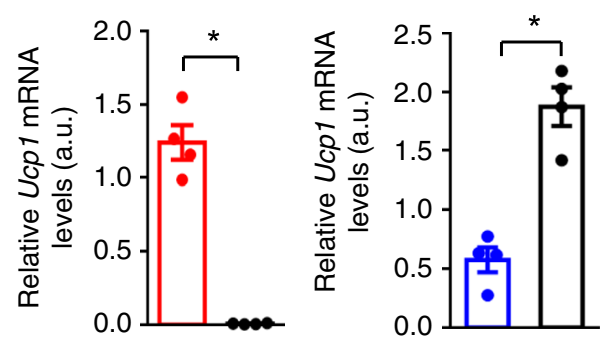

d
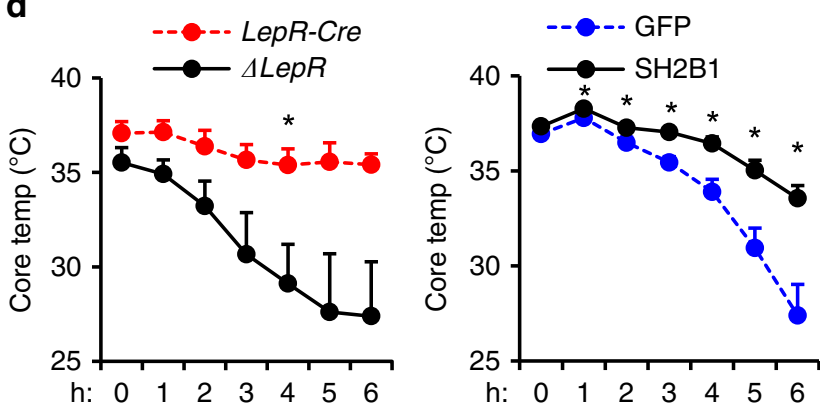

Fig. 5 Sh2b1 in LepR neurons supports the maintenance and function of BAT. a-c BAT was harvested from Sh2b1 1 LepR and $L e p R$-Cre males at 10 weeks of age, and from Sh2b1f/f males in 11 weeks after they were bilaterally injected into the MBH with AAV1-hSyn-Cre or AAV1-hSyn-GFP vectors (on chow diet). Adult C57BL/6J males were bilaterally injected into the MBH with AAV9-CAG-SH2B1 $\beta$ or AAV9-CAG-GFP vectors and then fed an HFD for 12 weeks. a Representative BAT images. Scale bar: $200 \mu \mathrm{m}$. Sh2b14LepR: $n=3$, LepR-Cre: $n=4$, AAV1-hSyn-Cre: $n=7$, AAV1-hSyn-GFP: $n=7$, AAV9-CAG-GFP: $n=$ 8, AAV9-CAG-SH2B1: $n=8$. b BAT extracts were immunoblotted with the indicated antibodies. Sh2b14LepR: $n=4$, LepR-Cre: $n=4$, AAV9-CAG-GFP: $n=4$ AAV9-CAG-SH2B1: $n=4$. c Ucp1 mRNA levels were normalized to 36B4 levels. Sh2b14LepR: $n=4$, LepR-Cre: $n=4$, AAV9-CAG-GFP: $n=4$, AAV9-CAG$\mathrm{SH} 2 \mathrm{~B} 1 \beta: n=4$. a.u. arbitrary units. $\mathbf{d}$ Core body temperature was recorded using E-Mitters in Sh2b $1^{\Delta L e p R}(n=3)$ and LepR-Cre $(n=3)$ at 10 weeks of age, on chow diet, and upon cold temperature $\left(4^{\circ} \mathrm{C}\right)$. Adult C57BL/6J males were bilaterally injected into the MBH with AAV9-CAG-GFP $(n=7)$ or AAV9-CAG$\mathrm{SH} 2 \mathrm{~B} 1 \beta(n=7)$ vectors and then fed an HFD for 10 weeks. Rectal temperature was measured upon cold exposure. Data are presented as mean \pm SEM. ${ }^{*} p<$ 0.05, two-tailed unpaired Student's $t$-test. Source data are provided as a Source Data file.

Sh $2 b 1$ in LepR neurons completely abrogated the ability of leptin to stimulate SNA in $S h 2 b 1^{\Delta L e p R}$ mice (Fig. 6f). These results indicate the Sh2b1 branch of LepR signaling pathways is required for leptin to stimulate the SNS.

We next set out to examine neuronal activity (c-Fos expression as a surrogate marker) in the central sympathetic network, focusing on the POA, DMH, ARC, paraventricular hypothalamus (PVH), and rostral raphe pallidus $(\mathrm{rRPa})$. These regions are known to control sympathetic outflows to $\mathrm{BAT}^{44}$. Cold exposure rapidly and robustly increased the number of c-Fos neurons in Sh2b1fff mice (Fig. 6g, h). Sh2b1 deficiency substantially suppressed cold-stimulated neuronal activation in the POA, DMH, and rRPa of $S h 2 b 1^{\Delta L e p R}$ mice (Fig. $6 \mathrm{~g}$, h). In the ARC, neural activity was significantly lower in $\operatorname{Sh} 2 b 1^{\Delta L e p R}$ relative to $S h 2 b 1^{f / f}$ mice at both 22 and $4^{\circ} \mathrm{C}$ (Fig. 6h).

To further confirm the role of LepR neuron Sh2b1 in regulating the SNS, we measured the levels of tyrosine hydroxylase (TH), a sympathetic nerve marker, in BAT. We previously validated that anti-TH antibody specifically recognizes $\mathrm{TH}$ in immunostaining ${ }^{45}$. Immunoreactivity to $\mathrm{TH}$ was dramatically lower in $\operatorname{Sh} 2 b 1^{\Delta L e p R}$ relative to LepR-Cre mice (Fig. 5a). Likewise, TH levels in BAT were also markedly reduced by $\mathrm{MBH}$-specific ablation of Sh2b1 (AAV-Cre vs AAV-GFP groups) (Fig. 5a). Conversely, $\mathrm{MBH}-$ specific overexpression of SH2B1 increased BAT TH levels (AAV$\mathrm{SH} 2 \mathrm{~B} 1$ vs AAV-GFP) (Fig. 5a). Collectively, these results unveil an 
a

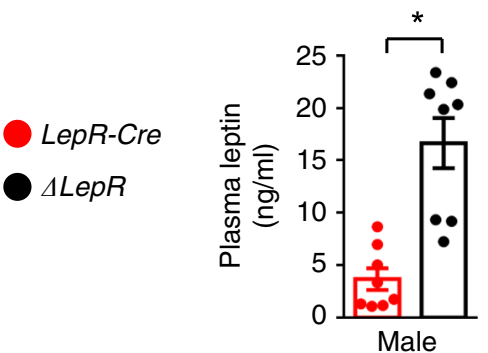

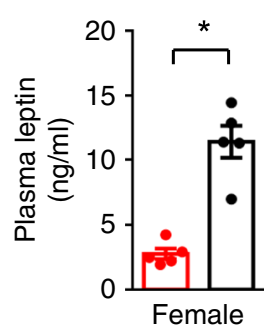

C

b
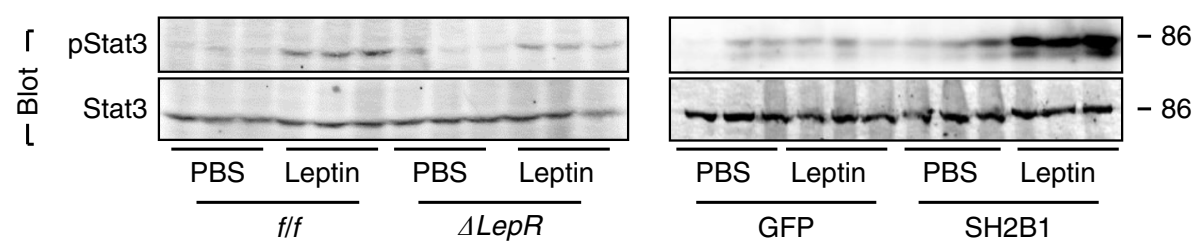

d Baseline Leptin (4 h)

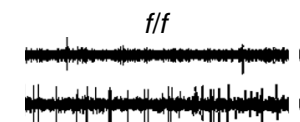

$\Delta L e p R$

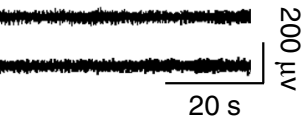

e

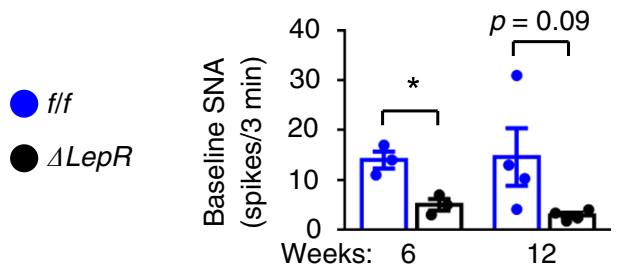

f

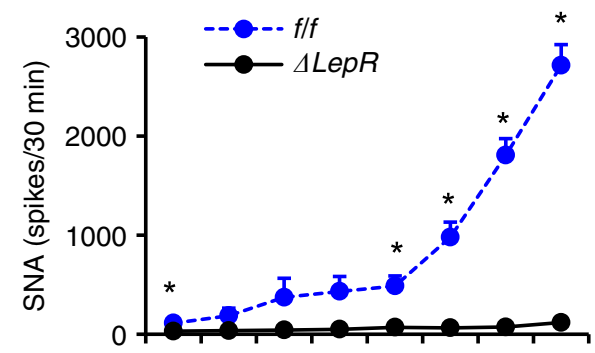

Leptin (h): $\quad \begin{array}{lllllllll}0.5 & 1.0 & 1.5 & 2.0 & 2.5 & 3.0 & 3.5 & 4.0\end{array}$

g $\quad 22^{\circ} \mathrm{C}$ $4{ }^{\circ} \mathrm{C}$

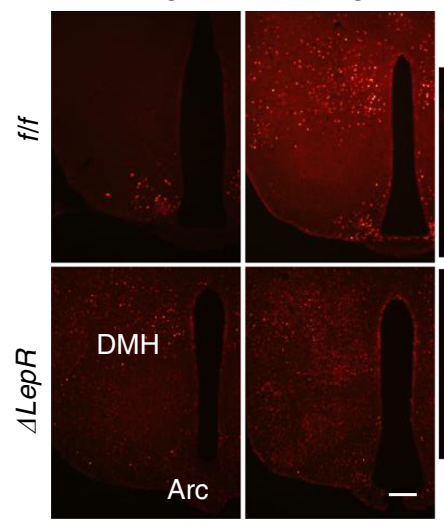

$22^{\circ} \mathrm{C}$

$4{ }^{\circ} \mathrm{C}$

$22^{\circ} \mathrm{C}$

$4^{\circ} \mathrm{C}$
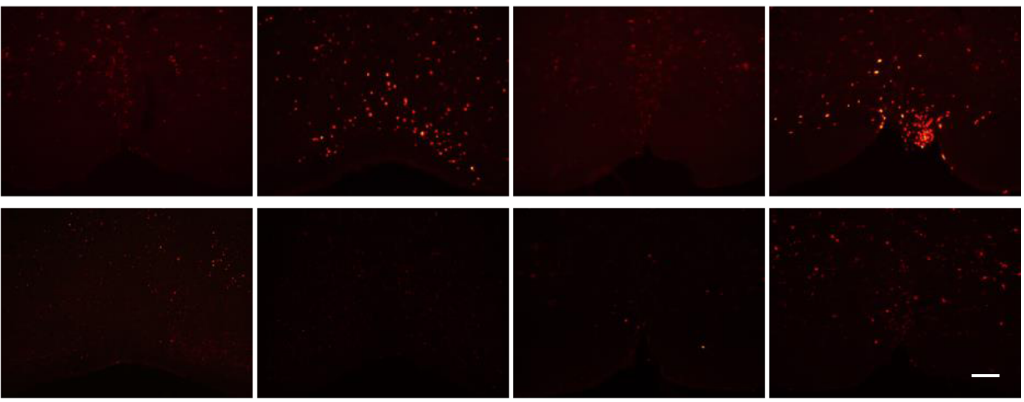

POA

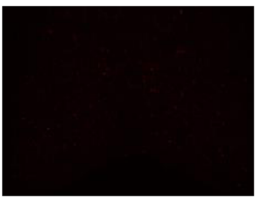

POA

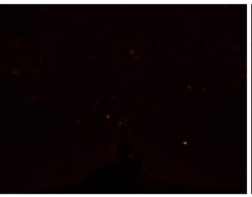

$\mathrm{rRPa}$

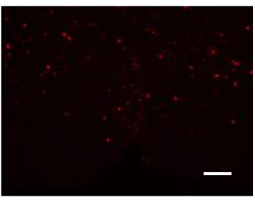

h

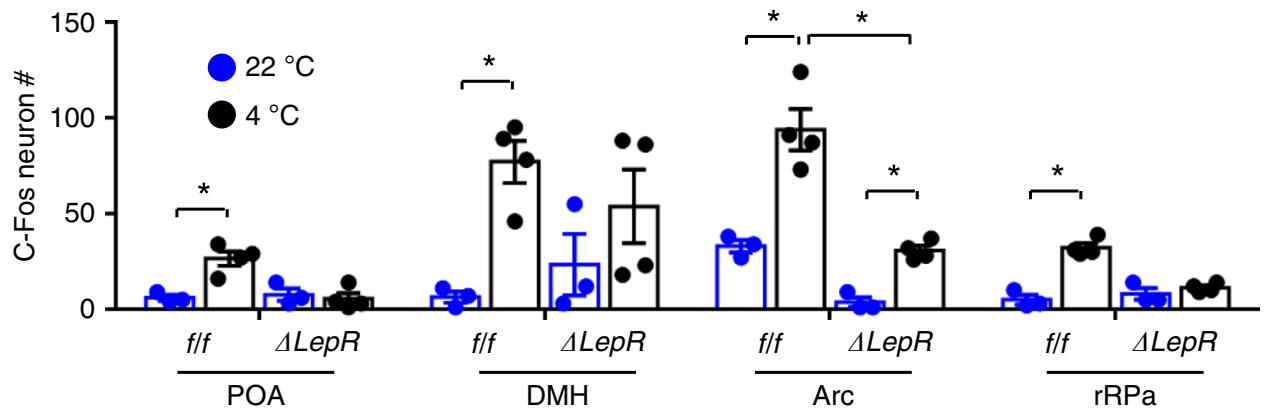

unrecognized Sh2b1/hypothalamic sympathetic network/SNS/ BAT energy expenditure axis.

prompting us to examine age-associated SNS degeneration. BAT $\mathrm{TH}$ levels were normal in young $S h 2 b 1^{\Delta L e p R}$ mice prior to 8 weeks of age (Fig. 7a, b), indicating that Sh2b1 in LepR neurons is dispensable for the development of adipose SNS. TH levels decreased progressively post 8 weeks of age and were barely

LepR neuron Sh2b1 preserves adipose SNS integrity in aging. $\operatorname{Sh} 2 b 1^{\Delta L e p R}$ mice develop obesity in an age-dependent manner, 
Fig. 6 Sh2b1 in LepR neurons mediates leptin stimulation of adipose SNS. a Overnight fasting plasma leptin levels at 22 weeks of age. Male: LepR-Cre: $n=8$, Sh $2 b 1^{\Delta L e p R:} n=8$; female: LepR-Cre: $n=5$, Sh $2 b 1^{\Delta L e p R}: n=5 . \mathbf{b}$, c $S h 2 b 7^{\Delta L e p R}$ and Sh2b1f/f males (6 weeks) were treated with leptin. Hypothalamic extracts were immunoblotted with antibodies against phospho-Stat3 (pTyr705) or Stat3. Stat3 phosphorylation was normalized to total Stat3 levels. Adult C57BL/6J males were bilaterally injected into the MBH with AAV9-CAG-SH2B1 $\beta$ or AAV9-CAG-GFP vectors, fed an HFD for 10 weeks, fasted overnight, and centrally injected with leptin for 15 min. Phosphorylation of hypothalamic Stat3 was assessed. $\mathbf{c} n=3$ per group. a.u. arbitrary units. d-f BAT sympathetic nerve activity (SNA). d Representative traces. e Baseline SNA at 6 ( $n=3$ mice per group) and 12 ( $n=4$ mice per group) weeks of age. $\mathbf{f}$ Time

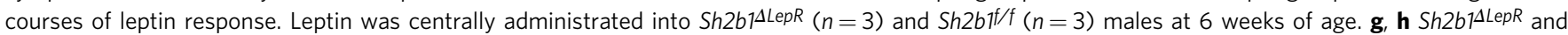
Sh $2 b 1^{t / f}$ males ( 7 weeks) were exposed to 22 or $4^{\circ} \mathrm{C}$ for $4 \mathrm{~h}$. Hypothalamic sections were stained with anti-Fos antibody. $\mathbf{g}$ Representative c-Fos staining of hypothalamic sections. Scale bar: $200 \mu \mathrm{m}$. h Number of c-Fos neurons in different hypothalamic areas. $4^{\circ} \mathrm{C}: n=4$ mice per group, $22^{\circ} \mathrm{C}: n=3$ mice per group. Data are presented as mean \pm SEM. ${ }^{\star} p<0.05$, two-tailed unpaired Student's $t$-test $(\mathbf{a}, \mathbf{c}, \mathbf{e}, \mathbf{f})$ or two-way ANOVA (h). Source data are provided as a Source Data file.

detectable in $S h 2 b 1^{\Delta L e p R}$ mice at 22 weeks of age (Fig. 7a, b). To confirm sympathetic degeneration, we assessed the levels of class III $\beta$-tubulin, a neuronal marker, using antibody TUJ1. TUJ1 immunoreactivity in BAT was abundant in $S h 2 b 1^{\mathrm{f} / f}$ but not Sh $2 b 1^{\Delta L e p R}$ mice at 22 weeks of age (Fig. 7c). Of note, Ucp1 downregulation followed the course of SNS deterioration in Sh2b1 $1^{\Delta L e p R}$ mice (Fig. $7 \mathrm{~d}$ ).

Next, we asked whether Sh2b1 deficiency in LepR neurons worsens SNS degeneration in white adipose tissue (WAT). Because sympathetic innervation of WAT is sparse and difficult to be detected ${ }^{46,47}$, we assessed phosphorylation of hormonesensitive lipase (HSL), a surrogate marker for SNS activation. HSL phosphorylation (pSer563 and pSer660) was normal or slightly higher in $S h 2 b 1^{\Delta L e p R}$ mice at 3 weeks of age; thereafter, HSL phosphorylation decreased progressively and became barely detectable at 22 weeks of age (Fig. 7e). Collectively, these results suggest that Sh2b1 in LepR neurons is involved in preserving the SNS in both BAT and WAT during aging.

Deletion of POMC neuron Sh2b1 is unable to induce obesity. We next aimed to further map Sh2b1 target neurons. Brain sections were prepared from wild type and global $S h 2 b 1$ knockout (negative control) mice and stained with anti-Sh2b1 antibody. Sh2b1 was detected in hypothalamic cells in wild-type mice (Supplementary Fig. 4a). To confirm these results, we generated Sh2b1-Cre knockin mice by inserting an IRES-eGFP-2A-Cre cassette into the Sh2b1 locus $3^{\prime}$ to the STOP codon (Supplementary Fig. 4b). GFP levels in Sh2b1-Cre mice were below detection thresholds. To facilitate detection of Sh2b1 neurons, Sh2b1-Cre drivers were crossed with Rosa-mTmG reporter mice to genetically label Sh2b1 neurons with mGFP in Sh2b1-Cre;Rosa-mTmG mice. We found that mGFP (a marker for expression of endogenous Sh2b1) was expressed in most of hypothalamic cells in Sh2b1-Cre;Rosa-mTmG but not Rosa-mTmG mice (Supplementary Fig. 4c). In line with these findings, Sh2b1 protein is detected in the entire brain by immunoblotting ${ }^{16}$. To confirm that proopiomelanocortin (POMC) and AgRP neurons express Sh2b1, hypothalamic sections were prepared from Sh2b1-Cre;mTmG mice and immunostained with antibodies to POMC and AgRP. Both POMC and AgRP neurons expressed mGFP (Supplementary Fig. 4d).

To explore the role of Sh2b1 in POMC neurons, we generated POMC neuron-specific Sh $2 b 1$ knockout (Sh $\left.2 b 1^{\triangle P O M C}\right)$ mice (Sh2b $1^{f / f} ; P O M C-C r e^{+/-}$) by crossing Sh2b ${ }^{f f f}$ mice with POMCCre drivers. Unlike Sh $2 b 1^{\triangle L e p R}$ mice, Sh $2 b 1^{\triangle P O M C}$ mice were grossly normal when fed a chow diet (Supplementary Fig. 5a). We placed Sh $2 b 1^{\Delta L e p R}$ mice on HFD for 10 weeks. Body weight, fat content, GTT, and ITT were comparable between $S h 2 b 1^{\Delta L e p R \text {, }}$ POMC-Cre, and Sh $2 b 1^{f / f}$ mice (Supplementary Fig. 5b-e). Together, these data suggest that POMC neuron-specific ablation of Sh2b1 is insufficient to induce obesity and metabolic disease.

\section{Discussion}

We herein identify LepR neurons as key Sh2b1 targets that mediate Sh2b1 protection against obesity, type 2 diabetes, and NAFLD. We demonstrated that LepR neuron-specific deletion of $\operatorname{Sh} 2 b 1$, or adult-onset deletion of $\operatorname{Sh} 2 b 1$ in the hypothalamus (containing LepR neurons), resulted in severe obesity, insulin resistance, and liver steatosis. Conversely, $\mathrm{MBH}$-specific overexpression of $\mathrm{SH} 2 \mathrm{~B} 1$ ameliorated HFD-induced obesity and metabolic syndromes. Leptin stimulation of the hypothalamic JAK2/Stat3 pathway was impaired in $S h 2 b 1^{\Delta L e p R}$ mice, supporting the notion that Sh2b1 is an endogenous sensitizer for leptin action, perhaps by enhancing JAK2 activation. Remarkably, ablation of Sh2b1 in LepR neurons abrogated the ability of leptin to stimulate sympathetic nerves projecting to BAT. Likewise, adult-onset, $\mathrm{MBH}$-specific ablation of Sh2b1 also impaired sympathetic transmissions in BAT. BAT became whitening and impaired in adaptive thermogenesis in both $S h 2 b 1^{\Delta L e p R}$ mice and mice with $\mathrm{MBH}$-specific ablation of Sh2b1, presumably owing to adipose SNS-deficits. Consequently, core body temperature was low and cold tolerance was impaired in both $S h 2 b 1^{\Delta L e p R}$ mice and $\mathrm{MBH}$-specific Sh $2 b 1$ knockout mice. These findings define LepR neuron Sh2b1 as a critical central regulator of thermogenesis and body temperature. Thus, we unveil an unrecognized leptin/LepR neuron Sh2b1/SNS/BAT/thermogenesis/body temperature axis. However, we cannot exclude the possibility that hypothermic Sh2b1 may increase thermogenesis and body temperature by an additional leptin-independent mechanism. For instance, Sh2b1 may enhance the ability of interleukin-6, a wellknown pyrogenic cytokine, to increase thermogenesis and body temperature through enhancing the JAK2/Stat3 pathway. Furthermore, hypothalamic Sh2b1 may increase body temperature by a SNS-independent mechanism, perhaps by enhancing the ability of hypothalamic-pituitary-thyroid axis to increase thermogenesis and body temperature. Given that the SNS/BAT pathway increases energy expenditure and weight loss, the LepR neuron Sh2b1/SNS/BAT/thermogenesis pathway is expected to mediate leptin stimulation of energy expenditure. Notably, food intake was relatively normal in $\operatorname{Sh} 2 b 1^{\Delta L e p R}$ mice, raising the possibility that leptin may regulate food intake and energy expenditure by Sh2b1-independent and Sh2b1-dependent pathways, respectively.

Both aging and obesity are associated with impaired adipose sympathetic nerve transmissions ${ }^{48-50}$, but the underlying mechanism is poorly understood. We found that BAT sympathetic nerve fibers deteriorated age-dependently and became undetectable in $S h 2 b 1^{\Delta L e p R}$ mice after 22 weeks of age. Following the course of sympathetic nerve degeneration, BAT progressively lost Ucp1 expression and thermogenic capability. Age-associated sympathetic nerve degeneration also occurred in WAT in $\operatorname{Sh} 2 b 1^{\Delta L e p R}$ mice. Of note, BAT sympathetic innervation and transmission were normal in young $S h 2 b 1^{\Delta L e p R}$ mice prior to 8 weeks of age. These results suggest that LepR neuron Sh2b1 is 
a

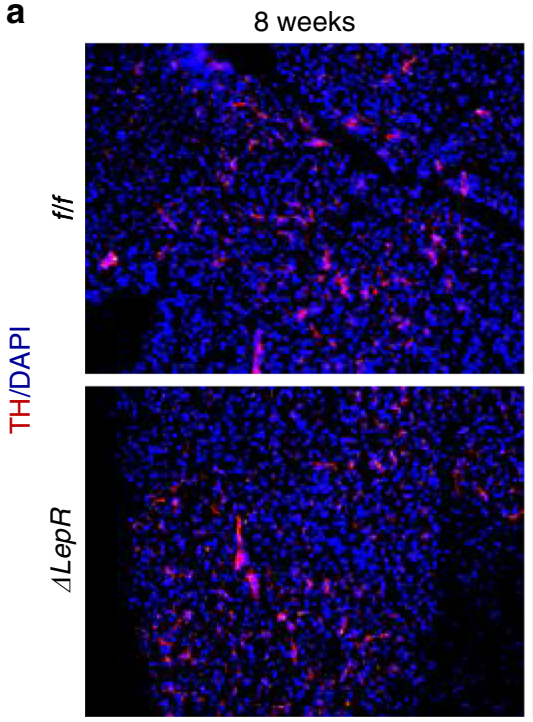

12 weeks
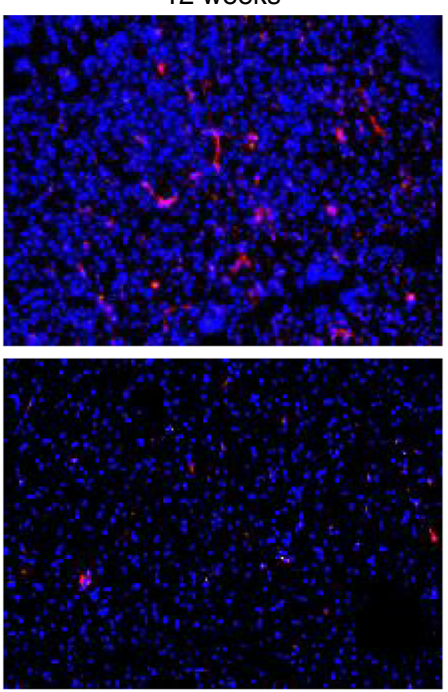

22 weeks
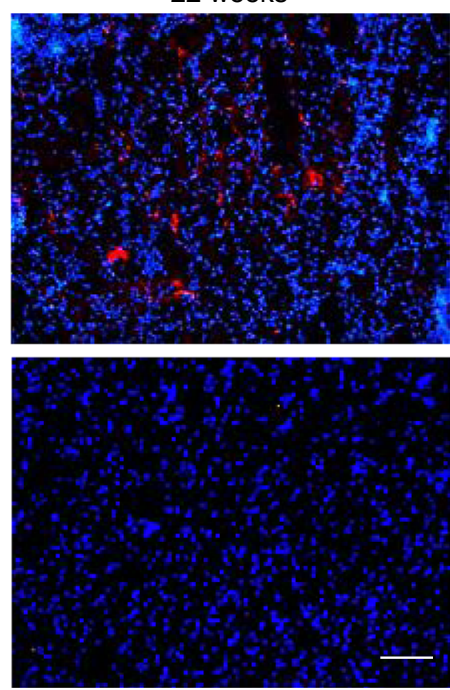

b

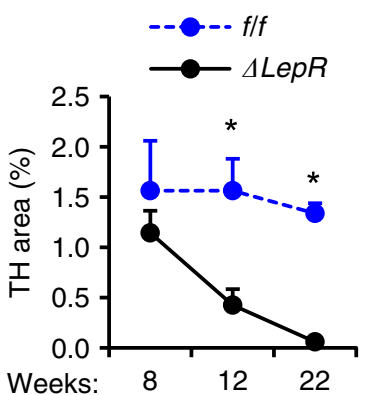

C

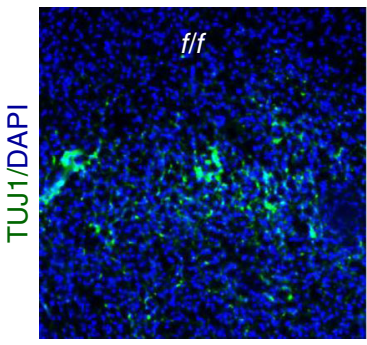

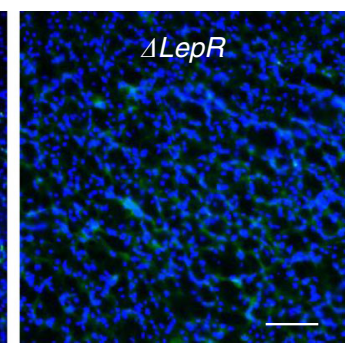

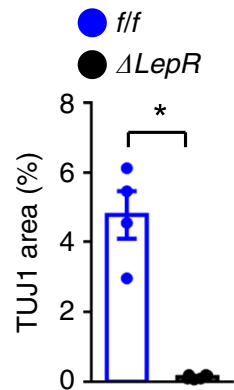

d
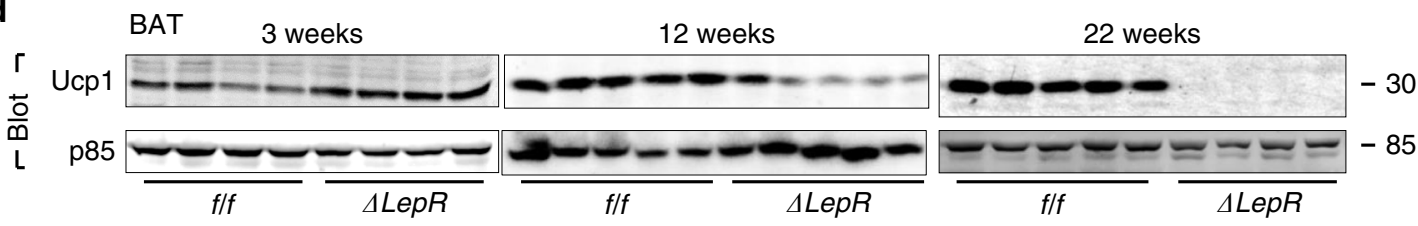

e
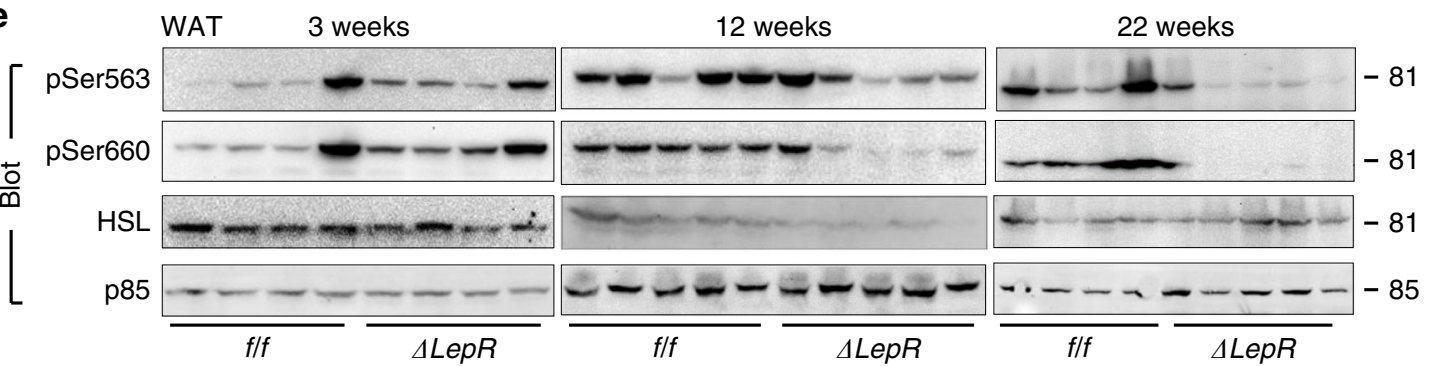

Fig. 7 Sh2b1 in LepR neurons supports the maintenance of adipose SNS. a, b BAT sections were prepared from male mice at 8,12 , and 22 weeks of age and stained with anti-tyrosine hydroxylase (TH) antibodies. a Representative images ( $n=3$ mice per group). $\mathbf{b}$ TH areas were quantified and normalized to total areas ( $n=3$ mice per group). c BAT sections were prepared at 22 weeks of age and stained with anti-TUJ1 antibody. TUJ1 areas were normalized to total areas. Sh2bif/f: $n=4$, Sh2blLLepR: $n=6$. d, e BAT (d) and epididymal WAT (e) extracts were prepared from male mice at 3, 12, and 22 weeks of age and immunoblotted with the indicated antibodies. Each lane represents an individual mouse. Data are presented as mean \pm SEM. ${ }^{\star} p<0.05$, two-tailed unpaired Student's t-test. Source data are provided as a Source Data file.

not required for adipose SNS development; rather, it plays an important role in preserving the adipose SNS against degeneration. Given that Sh2b1 is expressed broadly in the hypothalamus, it is not surprising that the neuronal activity of the central sympathetic network, particularly in the POA, DMH, and rRPa, is inhibited in Sh2b1 ${ }^{\Delta L e p R}$ mice. These results suggest that hypothalamic Sh2b1 preserves adipose SNS integrity and sympathetic transmissions by a top-down mechanism, perhaps by enhancing leptin and/or other hormone and neuropeptide signaling in the central sympathetic network. We acknowledge that our data do not exclude the possibility that adipose SNS deterioration may be secondary to obesity in $S h 2 b 1^{\Delta L e p R}$ mice. Additional studies are warranted to further characterize hypothalamic Sh2b1 circuits that protect against adipose SNS degeneration. 
The leptin resistance phenotype of $S h 2 b 1^{\Delta L e p R}$ mice supports a concept that Sh2b1 is an endogenous enhancer of leptin sensitivity in vivo. Strikingly, ablation of Sh2b1 in LepR neurons abolished leptin stimulation of the adipose SNS, indicating that Sh2b1 mediates leptin actions on the SNS and energy expenditure. We acknowledge that we did not detect significant difference in energy expenditure (normalized to mice) between $S h 2 b 1^{\Delta L e p R}$ and Sh2b1ff mice. We also did not observe the RER circadian rhythm. We speculate that assay-related stress and/or other factors may influence energy expenditure and the circadian rhythm, thereby masking difference between these two groups. Notably, the hypothalamic PI 3-kinase pathway was reported to mediate leptin stimulation of the SNS ${ }^{51,52}$. Sh2b1 mediates leptin stimulation of the PI 3-kinase pathway by recruiting IRS proteins to JAK2 ${ }^{13}$, raising the possibility that the LepRb/Sh2b1/PI 3-kinase pathway may mediate leptin stimulation of the SNS/BAT/thermogenesis axis. However, we do not exclude the possibility that Sh2b1 in LepR neurons may promote SNS activity and maintenance by additional mechanisms. For instance, Sh2b1 enhances BDNF and insulin signaling in cell cultures ${ }^{16-18,53}$. BDNF and insulin, like leptin, also activate the SNS/BAT energy expenditure axis $^{16-18,54,55}$. Sh2b1 may increase the ability of insulin and/or BDNF to stimulate energy expenditure. Of note, deletion of neither $S h 2 b 2$ nor $S h 2 b 3$ results in obesity ${ }^{56,57}$, suggesting that the Sh2b1 actions on energy balance and body weight cannot be replaced by other Sh $2 \mathrm{~b}$ family members.

We found that Sh2b1 was widely expressed in the entire brain, including hypothalamic POMC neurons and AgRP neurons. $S h 2 b 1^{\triangle P O M C}$ mice with POMC neuron-specific ablation of Sh2b1, unlike $S h 2 b 1^{\Delta L e p R}$ mice, did not develop obesity and insulin resistance. These results are not unexpected, considering that ablation of LepR in POMC neurons, AgRP neurons, or both only slightly affects body weight and metabolism ${ }^{58,59}$. Ablation of LepR in Ghrh, Htr2c, or Prlh neurons also fails to cause obesity ${ }^{60,61}$. We postulate that Sh2b1 deficiency in POMC neurons may activate a compensatory mechanism in other Sh2b1 subpopulations, which masks the action of POMC neuron Sh2b1 on body weight and metabolism.

In conclusion, we unravel an unrecognized LepR neuron Sh2b1/SNS/adipose energy expenditure axis that combats against obesity, type 2 diabetes, and NAFLD. LepR neuron Sh2b1 mediates leptin stimulation of the SNS and supports preservation of adipose SNS against degeneration. The Sh2b1/SNS/fat axis may serve as a potential therapeutic target for the treatment of obesity and metabolic disease.

\section{Methods}

Animals. Sh $2 b 1^{f / f}$ and LepR-Cre (knockin of Cre in the LepR $3^{\prime} \mathrm{UTR}$ ) mice (C57BL/ 6 background) were characterized previously ${ }^{40,62}$. Sh $2 b 1^{f / f}$ mice were crossed with LepR-Cre mice to generate Sh $2 b 1^{\Delta L e p R}$ mice $\left(S h 2 b 1^{f / f} ; C r e^{+/+}\right)$. Because Cre levels in LepR-Cre $e^{+/-}$mice are insufficient to delete target genes ${ }^{41,63,64}$, we generated homozygous LepR-Cre ${ }^{+/+}$mice to delete Sh $2 b 1$. A target vector containing an IRES-EGFP-2A-Cre fragment at the Sh $2 b 1$ locus ( $3^{\prime}$ to the STOP codon) was used to generate $\mathrm{Sh} 2 \mathrm{bl}$-Cre knockin mice through ES cell homologous recombination (Cyagen Biosciences Inc., Santa Clara, CA). Rosa26- $m$ TmG reporter mice were from the Jackson Laboratory (007676). Mice were housed on a $12 \mathrm{~h}$ light-dark cycle and fed ad libitum either a normal chow diet ( $9 \%$ fat; TestDiet, St. Louis, MO) or an HFD (60\% fat; Research Diets, New Brunswick, NJ).

Ethics statements. Animal research was complied with all relevant ethnic regulations. Animal experiments were conducted following the protocols approved by the University of Michigan Institutional Animal Care and Use Committee (IACUC).

Stereotaxic microinjection. Mice were anesthetized with isoflurane and mounted on an Ultra Precise Small Animal Stereotaxic Alignment System (Model 963, David KOPF Instruments, Tujunga, CA). Body temperature was maintained within a normal range during the entire procedures using a thermal pad. The skull was exposed to identify the bregma and lambda, and a small opening in the skull was made using a bone drill at coordinates $(\mathrm{mm}):-1.5(\mathrm{a}-\mathrm{p}), \pm 0.4(\mathrm{~m}-\mathrm{l})$, and -5.8 $(\mathrm{d}-\mathrm{v})$. AAV vectors $(0.5 \mu \mathrm{l})$ were injected into the hypothalamus in $10 \mathrm{~min}$ using UltraMicroPumps with SYS-Micro4 Controller (UMP3-2, World Precision Instruments Inc, Sarasota, FL). AAV1.hSyn.HI.eGFP-Cre and AAV1.hSyn.eGFP (control) were purchased from the Penn Vector Core, University of Pennsylvania School of Medicine. Cre expression is under the control of the neuron-specific human synapsin promoter. We prepared AAV9-CAG-SH2B1 and AAV9-CAG GFP (control) vectors in which SH2B1 $\beta$ expression is under the control of the constitutively active synthetic $C A G$ promoter.

Body core temperature and locomotor activity. Mice were anesthetized with isoflurane, and a G2 E-Mitter (870-0010-01, Starr life Sciences Corp, Oakmont, PA) was surgically implanted in the abdominal cavity. Locomotor activity and core body temperature were monitored using ER-4000 Energizer/Receiver (Bend, OR). Data were analyzed using Vitalview software (Starr life Sciences Corp,

Oakmont, PA).

Plasma insulin and leptin, GTT, and ITT. Blood samples were collected from tail veins. Plasma insulin and leptin were measured using insulin and leptin ELISA kits (CRYSTAL CHEM, Downers Grove, IL), respectively. For GTT, mice were fasted for $16 \mathrm{~h}$ and intraperitoneally injected with glucose ( $2 \mathrm{~g} / \mathrm{kg}$ body weight), and blood glucose was measured in $0,15,30,60$, and $120 \mathrm{~min}$ after injection. For ITT, mice were fasted for $6 \mathrm{~h}$ and intraperitoneally injected with insulin $(0.7 \mathrm{U} / \mathrm{kg}$ for $S h 2 b 1^{\Delta L e p R}$ mice, $0.6 \mathrm{U} / \mathrm{kg}$ for AAV-Cre or AAV-SH2B1 $\beta$ transduced mice), and blood glucose was measured in $0,15,30$, and $60 \mathrm{~min}$ after injection.

Fat content and energy expenditure. Fat content and lean body mass were measured using a dual-energy X-ray absorptiometry pDexa (Norland Stratec). Heads, neck, and tails were not included in the pDexa analysis. Energy expenditure was measured by indirect calorimetry (Windows Oxymax Equal Flow System, Columbus Instruments, Columbus, $\mathrm{OH}$ ).

Body composition and liver TAG levels. Mice were euthanized and organs wer harvested and weighted. Liver samples were homogenized in $1 \%$ acetic acid and extracted using chloroform:methanol (2:1). The organic phase was dried via evaporation and dissolved in isopropanol. TAG levels were measured using a TAG assay kit (Pointe Scientific Inc., Canton, MI) and normalized to liver weight.

Immunostaining. Brain and BAT sections were prepared using a Leica cryostat (Leica Biosystems Nussloch GmbH, Nussloch, Germany), and immunostained with the indicated antibodies (Supplemental Table 1). Images were visualized using a BX51 Microscope (Olympus, Tokyo, Japan) and a DP72 digital camera (Olympus, Tokyo, Japan).

Immunoblotting. Mice were fasted overnight and injected with insulin, and liver and skeletal muscle were harvested $5 \mathrm{~min}$ later. $S h 2 b 1^{\Delta L e p R}$ and $S h 2 b 1^{f / f}$ males ( 6 weeks) were treated with leptin $(1 \mathrm{mg} / \mathrm{kg}$ body weight, ip), and hypothalami were harvested $45 \mathrm{~min}$ later. Tissues were homogenized in ice-cold lysis buffer (50 mM Tris HCl, pH 7.5, 0.5\% Nonidet P-40, 150 mM NaCl, 2 mM EGTA, 1 mM $\mathrm{Na}_{3} \mathrm{VO}_{4}, 100 \mathrm{mM} \mathrm{NaF}, 10 \mathrm{mM} \mathrm{Na}{ }_{4} \mathrm{P}_{2} \mathrm{O}_{7}, 1 \mathrm{mM}$ phenylmethylsulfonyl fluoride, $10 \mu \mathrm{g} / \mathrm{ml}$ aprotinin, $10 \mu \mathrm{g} / \mathrm{ml}$ leupeptin). Tissue extracts were immunoblotted with the indicated antibodies (Supplemental Table 1).

qPCR. qPCR was performed using Radiant ${ }^{\mathrm{m}}$ SYBR Green $2 \times$ Lo-ROX qPCR Kits (Alkali Scientific, Pompano Beach, FL) and StepOnePlus RT PCR Systems (Life Technologies Corporation, NY, USA). Primers Ucp1-F: ATACTGGCAGATGA CGTCCC, Ucp1-R: GTACATGGACATCGCACAGC; 36B4-F: AAGCGCGTCCT GGCATTGTCT, 36B4-R: CCGCAGGGGCAGCAGTGGT.

Electrophysiological recordings. Mice (5 or 11 weeks) were implanted with intracerebroventricular (icv) cannula and subjected to recordings after a 1-week recover period. Mice were anesthetized with ketamine $(29 \mathrm{mg} / \mathrm{kg}$ body weight, intramuscular). BAT sympathetic nerve fibers were recorded using AxoScope 10.2 (San Jose, CA). Leptin $(0.5 \mu \mathrm{l} / \mathrm{mouse}, 1 \mathrm{mg} / \mathrm{ml})$ was injected via icv cannula. Nerve activity was amplified (NL104), filtered (NL 125/126, Neurolog, Digitimer Ltd), passed 100-1000 Hz, digitized (CED 1401, Cambridge Electronic Design, Cambridge, UK), and analyzed offline using Spike2 software (Cambridge Electronic Design, Cambridge, UK). To quantify SNA, the number of action potentials crossing a pre-set threshold was determined per second. The threshold was calculated at twice the baseline nerve activity for all experiments.

Statistical analysis. Data were presented as means \pm SEM. Differences between two groups were analyzed with two-tailed Student's $t$-test, and differences between more than two groups were analyzed using one-way and two-way analysis of variance (ANOVA) and Bonferroni posttest using GraphPad Prism 7. A P value less than 0.05 was considered significant. 
Reporting summary. Further information on research design is available in the Nature Research Reporting Summary linked to this article.

\section{Data availability}

The authors declare that the data supporting the findings of this study are available within the paper and supplementary information files. The source data underlying Figs. 1a, 2a-d, 6d, h and 7c and Supplementary Figs 1a and 5d are provided as a Source Data file.

Received: 9 November 2018; Accepted: 3 March 2020;

Published online: 23 March 2020

\section{References}

1. Zhou, Y. \& Rui, L. Leptin signaling and leptin resistance. Front. Med. 7, 207-222 (2013).

2. Patterson, C. M., Leshan, R. L., Jones, J. C. \& Myers, M. G. Jr. Molecular mapping of mouse brain regions innervated by leptin receptor-expressing cells. Brain Res. 1378, 18-28 (2011).

3. Scott, M. M. et al. Leptin targets in the mouse brain. J. Comp. Neurol. 514, 518-532 (2009).

4. Morris, D. L. \& Rui, L. Recent advances in understanding leptin signaling and leptin resistance. Am. J. Physiol. Endocrinol. Metab. 297, E1247-E1259 (2009).

5. Mori, H. et al. Socs 3 deficiency in the brain elevates leptin sensitivity and confers resistance to diet-induced obesity. Nat. Med. 10, 739-743 (2004).

6. Howard, J. K. et al. Enhanced leptin sensitivity and attenuation of dietinduced obesity in mice with haploinsufficiency of Socs3. Nat. Med. 10, 734-738 (2004).

7. Matarazzo, V. et al. Inactivation of Socs 3 in the hypothalamus enhances the hindbrain response to endogenous satiety signals via oxytocin signaling. J. Neurosci. 32, 17097-17107 (2012).

8. Zabolotny, J. M. et al. PTP1B regulates leptin signal transduction in vivo. Dev. Cell 2, 489-495 (2002).

9. Cheng, A. et al. Attenuation of leptin action and regulation of obesity by protein tyrosine phosphatase 1B. Dev. Cell 2, 497-503 (2002).

10. Bence, K. K. et al. Neuronal PTP1B regulates body weight, adiposity and leptin action. Nat. Med. 12, 917-924 (2006)

11. Rousso-Noori, L. et al. Protein tyrosine phosphatase epsilon affects body weight by downregulating leptin signaling in a phosphorylation-dependent manner. Cell Metab. 13, 562-572 (2011).

12. Loh, K. et al. Elevated hypothalamic TCPTP in obesity contributes to cellular leptin resistance. Cell Metab. 14, 684-699 (2011).

13. Duan, C., Li, M. \& Rui, L. SH2-B promotes insulin receptor substrate 1 (IRS1)- and IRS2-mediated activation of the phosphatidylinositol 3-kinase pathway in response to leptin. J. Biol. Chem. 279, 43684-43691 (2004).

14. Li, Z., Zhou, Y., Carter-Su, C., Myers, M. G. Jr. \& Rui, L. SH2B1 enhances leptin signaling by both Janus kinase 2 Tyr813 phosphorylation-dependent and -independent mechanisms. Mol. Endocrinol. 21, 2270-2281 (2007).

15. Rui, L. \& Carter-Su, C. Identification of SH2-bbeta as a potent cytoplasmic activator of the tyrosine kinase Janus kinase 2. Proc. Natl. Acad. Sci. USA 96 , 7172-7177 (1999).

16. Jiang, L. et al. Neural deletion of Sh2b1 results in brain growth retardation and reactive aggression. FASEB J. 32, 1830-1840 (2018).

17. Shih, C. H., Chen, C. J. \& Chen, L. New function of the adaptor protein SH2B1 in brain-derived neurotrophic factor-induced neurite outgrowth. PLoS ONE $\mathbf{8}$, e79619 (2013).

18. Morris, D. L., Cho, K. W., Zhou, Y. \& Rui, L. SH2B1 enhances insulin sensitivity by both stimulating the insulin receptor and inhibiting tyrosine dephosphorylation of insulin receptor substrate proteins. Diabetes $\mathbf{5 8}$, 2039-2047 (2009).

19. Rui, L. \& Carter-Su, C. Platelet-derived growth factor (PDGF) stimulates the association of SH2-Bbeta with PDGF receptor and phosphorylation of SH2Bbeta. J. Biol. Chem. 273, 21239-21245 (1998).

20. Qian, X., Riccio, A., Zhang, Y. \& Ginty, D. D. Identification and characterization of novel substrates of Trk receptors in developing neurons. Neuron 21, 1017-1029 (1998).

21. Rui, L., Herrington, J. \& Carter-Su, C. SH2-B is required for nerve growth factor-induced neuronal differentiation. J. Biol. Chem. 274, 10590-10594 (1999).

22. Li, M., Li, Z., Morris, D. L. \& Rui, L. Identification of SH2B2beta as an inhibitor for SH2B1- and SH2B2alpha-promoted Janus kinase-2 activation and insulin signaling. Endocrinology 148, 1615-1621 (2007).

23. Duan, C., Yang, H., White, M. F. \& Rui, L. Disruption of the SH2-B gene causes age-dependent insulin resistance and glucose intolerance. Mol. Cell Biol. 24, 7435-7443 (2004).
24. Ren, D., Li, M., Duan, C. \& Rui, L. Identification of SH2-B as a key regulator of leptin sensitivity, energy balance, and body weight in mice. Cell Metab. 2, 95-104 (2005).

25. Song, W. et al. SH2B regulation of growth, metabolism, and longevity in both insects and mammals. Cell Metab. 11, 427-437 (2010).

26. Willer, C. J. et al. Six new loci associated with body mass index highlight a neuronal influence on body weight regulation. Nat. Genet. 41, 25-34 (2009).

27. Thorleifsson, G. et al. Genome-wide association yields new sequence variants at seven loci that associate with measures of obesity. Nat. Genet. 41, 18-24 (2009).

28. Rui, L. SH2B1 regulation of energy balance, body weight, and glucose metabolism. World J. Diabetes 5, 511-526 (2014).

29. Bochukova, E. G. et al. Large, rare chromosomal deletions associated with severe early-onset obesity. Nature 463, 666-670 (2010).

30. Walters, R. G. et al. A new highly penetrant form of obesity due to deletions on chromosome 16p11.2. Nature 463, 671-675 (2010).

31. Bachmann-Gagescu, R. et al. Recurrent 200-kb deletions of $16 \mathrm{p} 11.2$ that include the $\mathrm{SH} 2 \mathrm{~B} 1$ gene are associated with developmental delay and obesity. Genet. Med. 12, 641-647 (2010).

32. Tabet, A. C. et al. Autism multiplex family with $16 \mathrm{p} 11.2 \mathrm{p} 12.2$ microduplication syndrome in monozygotic twins and distal 16p11.2 deletion in their brother. Eur. J. Hum. Genet. 20, 540-546 (2012).

33. Doche, M. E. et al. Human SH2B1 mutations are associated with maladaptive behaviors and obesity. J. Clin. Invest. 122, 4732-4736 (2012).

34. Foucan, L. et al. High prevalence of rare monogenic forms of obesity in obese Guadeloupean Afro-Caribbean children. J. Clin. Endocrinol. Metab. 103, 539-545 (2018).

35. Volckmar, A. L. et al. Mutation screen in the GWAS derived obesity gene SH2B1 including functional analyses of detected variants. BMC Med. Genomics 5, 65 (2012)

36. Zheng, Z. et al. Screening for coding variants in FTO and SH2B1 genes in chinese patients with obesity. PLoS ONE 8, e67039 (2013).

37. Guo, A. et al. Immunoaffinity enrichment and mass spectrometry analysis of protein methylation. Mol. Cell Proteomics 13, 372-387 (2014).

38. Aerts, E. et al. Genetic and structural variation in the SH2B1 gene in the Belgian population. Mol. Genet. Metab. 115, 193-198 (2015).

39. Ren, D. et al. Neuronal SH2B1 is essential for controlling energy and glucose homeostasis. J. Clin. Invest 117, 397-406 (2007)

40. Leshan, R. L., Bjornholm, M., Munzberg, H. \& Myers, M. G. Jr. Leptin receptor signaling and action in the central nervous system. Obesity (Silver Spring) 14, 208S-212S (2006)

41. Sadagurski, M. et al. IRS2 signaling in LepR-b neurons suppresses FoxO1 to control energy balance independently of leptin action. Cell Metab. 15, 703-712 (2012).

42. Feldmann, H. M., Golozoubova, V., Cannon, B. \& Nedergaard, J. UCP1 ablation induces obesity and abolishes diet-induced thermogenesis in mice exempt from thermal stress by living at thermoneutrality. Cell Metab. 9 , 203-209 (2009)

43. Haynes, W. G., Morgan, D. A., Walsh, S. A., Mark, A. L. \& Sivitz, W. I. Receptor-mediated regional sympathetic nerve activation by leptin. J. Clin. Invest. 100, 270-278 (1997).

44. Morrison, S. F. Central neural control of thermoregulation and brown adipose tissue. Auton. Neurosci. 196, 14-24 (2016).

45. Shen, H., Jiang, L., Lin, J. D., Omary, M. B. \& Rui, L. Brown fat activation mitigates alcohol-induced liver steatosis and injury in mice. J. Clin. Invest. 130, 2305-2317 (2019).

46. Zeng, W. et al. Sympathetic neuro-adipose connections mediate leptin-driven lipolysis. Cell 163, 84-94 (2015).

47. Chi, J. et al. Three-dimensional adipose tissue imaging reveals regional variation in beige fat biogenesis and PRDM16-dependent sympathetic neurite density. Cell Metab. 27, 226-236 e223 (2018)

48. Coppack, S. W. et al. Whole body, adipose tissue, and forearm norepinephrine kinetics in lean and obese women. Am. J. Physiol. 275, E830-E834 (1998).

49. Dodt, C., Lonnroth, P., Fehm, H. L. \& Elam, M. The subcutaneous lipolytic response to regional neural stimulation is reduced in obese women. Diabetes 49, $1875-1879$ (2000)

50. Greaney, J. L., Kenney, W. L. \& Alexander, L. M. Sympathetic regulation during thermal stress in human aging and disease. Auton. Neurosci. 196, 81-90 (2016).

51. Harlan, S. M., Guo, D. F., Morgan, D. A., Fernandes-Santos, C. \& Rahmouni, K. Hypothalamic mTORC1 signaling controls sympathetic nerve activity and arterial pressure and mediates leptin effects. Cell Metab. 17, 599-606 (2013).

52. Buettner, C. et al. Leptin controls adipose tissue lipogenesis via central, STAT3-independent mechanisms. Nat. Med. 14, 667-675 (2008).

53. Ahmed, Z. \& Pillay, T. S. Functional effects of APS and SH2-B on insulin receptor signalling. Biochem. Soc. Trans. 29, 529-534 (2001)

54. Cao, L. et al. White to brown fat phenotypic switch induced by genetic and environmental activation of a hypothalamic-adipocyte axis. Cell Metab. 14, 324-338 (2011). 
55. Dodd, G. T. et al. Leptin and insulin act on POMC neurons to promote the browning of white fat. Cell 160, 88-104 (2015).

56. Li, M., Ren, D., Iseki, M., Takaki, S. \& Rui, L. Differential role of SH2-B and APS in regulating energy and glucose homeostasis. Endocrinology 147, 2163-2170 (2006).

57. Mori, T., Suzuki-Yamazaki, N. \& Takaki, S. Lnk/Sh2b3 regulates adipose inflammation and glucose tolerance through group 1 ILCs. Cell Rep. 24, 1830-1841 (2018).

58. Balthasar, N. et al. Leptin receptor signaling in POMC neurons is required for normal body weight homeostasis. Neuron 42, 983-991 (2004).

59. van de Wall, E. et al. Collective and individual functions of leptin receptor modulated neurons controlling metabolism and ingestion. Endocrinology 149, 1773-1785 (2008).

60. Rupp, A. C. et al. Specific subpopulations of hypothalamic leptin receptorexpressing neurons mediate the effects of early developmental leptin receptor deletion on energy balance. Mol. Metab. 14, 130-138 (2018).

61. Dodd, G. T. et al. The thermogenic effect of leptin is dependent on a distinct population of prolactin-releasing peptide neurons in the dorsomedial hypothalamus. Cell Metab. 20, 639-649 (2014).

62. Chen, Z., Morris, D. L., Jiang, L., Liu, Y. \& Rui, L. SH2B1 in beta-cells regulates glucose metabolism by promoting beta-cell survival and islet expansion. Diabetes 63, 585-595 (2014).

63. Leshan, R. L. et al. Direct innervation of GnRH neurons by metabolic- and sexual odorant-sensing leptin receptor neurons in the hypothalamic ventral premammillary nucleus. J. Neurosci. 29, 3138-3147 (2009).

64. Leinninger, G. M. et al. Leptin acts via leptin receptor-expressing lateral hypothalamic neurons to modulate the mesolimbic dopamine system and suppress feeding. Cell Metab. 10, 89-98 (2009).

\section{Acknowledgements}

We thank Ed Stunkel, Brian Pierchala, and Christin Carter-Su (University of Michigan) for helpful discussion and suggestions. This study was supported by grants RO1 DK094014, RO1 DK115646, DK-114220 (to L.R.), and R01 DK110436 and P30 DK34933 (to C.O.) from the National Institutes of Health and Grant \#1-18-IBS-189 from the American Diabetes Association (to L.R.). This work utilized the cores supported by the Michigan Diabetes Research and Training Center (NIH DK020572), Michigan Metabolomics and Obesity Center (DK089503), and the University of Michigan Gut Peptide Research Center (NIH DK34933).

\section{Author contributions}

L.J., H. Su, X.W., H. Shen, and M.-H.K. conducted the experiments, L.J. and L.R. designed the experiments and wrote the paper, and L.J., H. Su, X.W., H. Shen, M.-H.K., Y.L., M.G.M., C.O., and L.R. performed data analyses and edited the paper.

\section{Competing interests}

The authors declare no competing interests.

\section{Additional information}

Supplementary information is available for this paper at https://doi.org/10.1038/s41467020-15328-3.

Correspondence and requests for materials should be addressed to L.R.

Peer review information Nature Communications thanks Qingchun Tong and the other anonymous reviewer(s) for their contribution to the peer review of this work. Peer reviewer reports are available.

Reprints and permission information is available at http://www.nature.com/reprints

Publisher's note Springer Nature remains neutral with regard to jurisdictional claims in published maps and institutional affiliations.

cc (i) Open Access This article is licensed under a Creative Commons Attribution 4.0 International License, which permits use, sharing, adaptation, distribution and reproduction in any medium or format, as long as you give appropriate credit to the original author(s) and the source, provide a link to the Creative Commons license, and indicate if changes were made. The images or other third party material in this article are included in the article's Creative Commons license, unless indicated otherwise in a credit line to the material. If material is not included in the article's Creative Commons license and your intended use is not permitted by statutory regulation or exceeds the permitted use, you will need to obtain permission directly from the copyright holder. To view a copy of this license, visit http://creativecommons.org/ licenses/by/4.0/.

(c) The Author(s) 2020 